Ann. scient. Éc. Norm. Sup.,

$4^{\mathrm{e}}$ série, t. 31, 1998, p. 561 à 589.

\title{
ON IRREDUCIBILITY OF STANDARD MODULES FOR GENERIC REPRESENTATIONS
}

\author{
By William CASSELMAN AND FreydoON SHAHIDI $\left(^{*}\right)$
}

\begin{abstract}
In this paper the authors generalize a result of Vogan on irreducibility of standard modules for generic representations from real groups to $p$-adic ones whenever the inducing data is supercuspidal. They also prove injectivity for standard modules in this case. As applications, the authors prove a number of results relating the poles of intertwining operators and points of reducibility of induced representations to the poles of $L$-functions defined by the second author, modulo a conjecture on them whose validity for classical groups is also verified here. A result on certain real groups with applications in liftings of automorphic forms from classical groups to general linear groups via $L$-functions is also proved. (C) Elsevier, Paris
\end{abstract}

RÉsumÉ. - Dans cet article les auteurs généralisent un résultat de Vogan sur l'irréductibilité des modules standards pour les représentations génériques des groupes réels vers les groupes $p$-adiques quand l'induite est supercuspidale. Ils prouvent également l'injectivité pour les modules standards dans ce cas. Les auteurs en déduisent quelques résultats reliant les pôles des opérateurs d'entrelacement et les points d'irréductibilité des représentations induites, aux pôles des fonctions $L$ définies par le deuxième auteur, modulo la validité d'une conjecture que l'on vérifie ici pour les groupes classiques. On montre aussi un résultat sur certains groupes réels avec applications aux correspondances des formes automorphes des groupes classiques vers les groupes linéaires via les fonctions $L$. (c) Elsevier, Paris

\section{Introduction}

The purpose of this paper is to prove a number of results in representation theory and harmonic analysis of local groups, some of which have important consequences in the theory of automorphic forms.

More precisely, let $\mathbf{G}$ be a quasisplit connected reductive group over a local field $F$ of characteristic zero (real, complex, or $p$-adic) and let $\mathbf{B}=\mathbf{T U}$ be a Borel subgroup of $\mathbf{G}$, where $\mathbf{T}$ is a maximal torus of $\mathbf{B}$ and $\mathbf{U}$ is its unipotent radical. Let $\mathbf{A}_{0}$ be the maximal split torus of $\mathbf{T}$. Fix a parabolic subgroup $\mathbf{P}$ of $\mathbf{G}$ defined over $F$ with a Levi decomposition $\mathbf{P}=\mathbf{M N}$, with $\mathbf{T} \subset \mathbf{M}$ and $\mathbf{N} \subset \mathbf{U}$. Let $\sigma$ be an irreducible tempered representation of $M=\mathbf{M}(F)$ and choose $\nu \in \mathfrak{a}_{\mathfrak{C}}^{*}$, the complex dual of the real Lie algebra of the split component $\mathbf{A}$ of $\mathbf{M}$. (See Section 1). Let $I(\nu, \sigma)$ be the representation (unitarily) induced from $\nu$ and $\sigma$. Assume $\nu$ is in the positive Weyl chamber (Section 1). Then $I(\nu, \sigma)$ is called a standard module. Let $J(\nu, \sigma)$ be the (unique) Langlands quotient of $I(\nu, \sigma)$ (cf. $[4,17,28])$. Up to conjugation of the data $(\nu, \sigma)$, every irreducible

(*) Partially supported by NSF Grant DMS-9622585. 
admissible representation of $G=\mathbf{G}(F)$ is uniquely equivalent to a $J(\nu, \sigma)$. Moreover, every irreducible admissible generic (having a Whittaker model) representation of $G$ is a $J(\nu, \sigma)$ with $\sigma$ an irreducible generic tempered representation of $M$.

When $F=\mathbf{R}$, it was proved by Vogan in [37] that if $J(\nu, \sigma)$ is generic, then $I(\nu, \sigma)=J(\nu, \sigma)$, i.e. $I(\nu, \sigma)$ is irreducible. The aim of this paper is to address this question and related ones for $p$-adic groups. In fact, in this paper we prove (Theorems 2.2 and 3.4):

THEOREM 1. - Suppose $\sigma$ is an irreducible unitary generic supercuspidal representation of $M$ and fix $\nu \in \mathfrak{a}_{\mathrm{C}}^{*}$ in the positive Weyl chamber.

a) Assume $J(\nu, \sigma)$ is generic. Then $I(\nu, \sigma)=J(\nu, \sigma)$, i.e. $I(\nu, \sigma)$ is irreducible.

b) Suppose $J(\nu, \sigma)$ is not generic. Then the unique irreducible subspace of $I(\nu, \sigma)$ is generic.

Equivalently, irreducible generic subquotients of $I(\nu, \sigma)$ are subrepresentations and are therefore unique.

In the special case that $\mathbf{P}=\mathbf{B}$, i.e. is a minimal parabolic subgroup and $\sigma$ is an unramified quasicharacter of $M=T$, Part a) of Theorem 1 was proved in [2], [20], and [22], earlier, each using a different method. We refer to [3], [14], and [41] for $\mathbf{G}=G L_{n}$, but general tempered $\sigma$.

When $\mathbf{G}=S p_{2 n}$ or $S O_{2 n+1}$, part a) was recently proved in the generality of arbitrary standard modules by Goran Muic in a very interesting manuscript [42]. As is the case with our results, his proof is based on the theory of $L$-functions developed in [23].

Although the result stated above is only for inducing supercuspidal data, the theorem is expected to be true in the generality of every standard module if part $b$ ) is formulated as:

1) Irreducible generic constituents of standard modules are subrepresentations. (See the remark after Definition 3.1.)

We have called this statement the generalized injectivity conjecture (Conjecture 3.3) and it is clear that it implies part a), i.e.

2) Standard modules for generic representations are irreducible.

Theorem 3.4 then proves this for inducing supercuspidal representations.

To state some applications of injectivity (e.g. Theorem 5.1), one needs to discuss a conjecture (Conjecture 7.1 of [23]) whose validity also plays an important role in the proofs given here and [42].

To explain the conjecture, assume $\mathbf{P}$ is maximal. But $\sigma$ is any irreducible admissible generic representation of $\mathbf{M}$. If $r$ is the adjoint action of ${ }^{L} M$, the $L$-group of $\mathbf{M}$ on the Lie algebra ${ }^{L} \mathfrak{n}$ of the $L$-group of $\mathbf{N}$, then $r=\bigoplus_{i=1}^{m} r_{i}$, with $r_{i}$ 's ordered as in [23], i.e. according to the order of eigenvalues of ${ }^{L} A$ in ${ }^{L} \mathfrak{n}$. Finally, for each $i, 1 \leq i \leq m$, let $L\left(s, \sigma, r_{i}\right)$ be the local $L$-function attached to $\sigma$ and $r_{i}$ as in [23]. When $F=\mathbf{R}$, the $L$-functions are those of Artin (cf. $[1,17,18,24]$ ). (See Section 6 here and Theorem 3.5 and Section 7 of [23].) Conjecturc 1.1 then demands that each $L\left(s, \sigma, r_{i}\right)$ be holomorphic for $\operatorname{Re}(s)>0$ whenever $\sigma$ is tempered.The normalized intertwining operators then satisfy the last condition $R_{7}$ set forth by Arthur in [1], whenever normalization is as in $[19,23]$. This conjecture has been verified in many cases in [23], including when $m=1$ or $\sigma$ is supercuspidal, and the subject matter of Section 4 of the present paper is to prove it whenever $\mathbf{G}$ is of classical type. This includes all the quasisplit classical groups.

$4^{\mathrm{e}}$ SÉRIE - TOME $31-1998-\mathrm{N}^{\circ} 4$ 
Next, let $I(\nu, \sigma)$ be a standard module attached to a tempered representation $\sigma$ of $M$, where $\mathbf{P}=\mathbf{M N}$ is the corresponding standard parabolic subgroup. Denote by $\widetilde{w}_{0}$ the longest element in the Weyl group of $\mathbf{A}_{\mathbf{0}}$ in $\mathbf{G}$ modulo that of $\mathbf{A}_{0}$ in $\mathbf{M}$. Fix a representative $w_{0}$ for $\widetilde{w}_{0}$ in $G$. Let $A\left(\nu, \sigma, w_{0}\right)$ be the standard intertwining operator from $I(\nu, \sigma)$ into $I\left(w_{0}(\nu), w_{0}(\sigma)\right)$ (cf. Section 1). It is well defined since $\nu$ is in the positive Weyl chamber. But its continuation to all of $\mathfrak{a}_{\mathfrak{C}}^{*}$ may have poles and that is where the problem lies. Assume $\mathbf{P}$ is maximal. Let $\alpha$ be the unique simple root in $\mathbf{N}$. Set $\tilde{\rho}=\langle\rho, \alpha\rangle^{-1} \rho$, where $\rho$ is half the sum of roots in N. Fix $s \in \mathbb{C}$. We may take $\nu=s \widetilde{\alpha} \in \mathfrak{a}_{\mathrm{C}}^{*}$. Then $\operatorname{Re}(s)>0$ since $\nu$ is in the positive Weyl chamber. Consider the operator

$$
\prod_{i=1}^{m} L\left(i s, \tilde{\sigma}, r_{i}\right)^{-1} A\left(s \widetilde{\alpha}, \sigma, w_{0}\right)
$$

on $I(s \tilde{\alpha}, \sigma)$.

The homomorphy of (3) for all $s \in \mathbb{C}$ has important global consequences through normalization of intertwining operators and Eisenstein series (cf. [11], [15], [19], [21], [30], [31], [43]), and although at present we are unable to prove it in general, there are practical instances when this can be accomplished. In fact, our Theorem 5.1 proves the holomorphy of (3) on all of $\mathbb{C}$ under what we call injectivity (Definition 3.1) for all the corresponding rank one standard modules. (See the remark after Definition 3.1.)

One important instance when Theorem 5.1 can be applied is when $F=\mathbb{R}$ or $\mathbb{C}$, and $\mathbf{G}=S O_{2 n}$, the split even special orthogonal group of rank $n$ (Theorem 6.1). The case in hand has an important application in the project of lifting automorphic forms from classical groups to general linear groups as being pursued in $[11,30,31]$, using the converse theorem for $L$-functions [9].

To check that the hypothesis of Theorem 5.1 is satisfied, it is sufficient to prove that standard modules for $G L_{n}(\mathbb{R})$ satisfy injectivity, i.e. their irreducible subrepresentations, which turn out to be a single one, are all generic (large). A proof of this was communicated to us by Vogan. We would like to thank him for providing us with a proof and allowing us to include it here.

Vogan's proof is quite instructive. It relies on cohomological induction and is therefore algebraic. On the other hand, after communications with him, the authors realized that there is an analytic proof of Theorem 6.2 due to Jacquet and Shalika (Proposition 4.2 of [13]) which relies on the theory of canonical extensions of Harish-Chandra modules as developed by Casselman [6] and Wallach [40]. The existence of this second proof, which in spirit is closer to our approach in Section 3, was envisioned and communicated by the first author to several people, many years ago (cf. [7], for example).

Our final application, Proposition 5.3 (and 5.4), determines the points of reducibility for every $I(s \tilde{\alpha}, \sigma)$ in terms of poles of $L$-functions, but under the assumption of validity of (2). When $F=\mathbb{R}$, the assumption is already proved, and therefore Proposition 5.3 shows that points of reducibility for $I(s \tilde{\alpha}, \sigma), R e(s)>0$, are precisely poles of $\prod_{i=1}^{m} L\left(1-i s, \sigma, r_{i}\right)$, where $L$-functions are those of Artin, attached to $\sigma$ and $r_{i}$ by Langlands [1], [17], [18], [24].

Proof of Conjecture 1.1 for the classical groups, given in Section 4 here, relies to certain extent, on what cuspidal inducing data for discrete series are. Except for Lemmas 4.1 and 4.6 of [34], we have relied entirely on our own method to determine them and our results are quite parallel to those of Tadić [34], [35], [36]. The relation between our 
two methods must certainly be explored furthcr. (See Remarks $4.21,4.22$, and 4.23 here and [42].) Lemma 4.1 has also been verified independently by Y. Zhang in a work in progress, using results of Harish-Chandra and Silberger on special orbits (cf. [28]).

Finally, since the $L$-functions are supposed to remain the same for members of each tempered $L$-packet (Conjecture 9.4 of [23]), one must explore the possibility of extending such results to non-generic representations. The project which has been started in [10] and is aimed at developing similar results for non-generic representations may eventually provide us with some evidence in this direction.

The second author would like to thank Joachim Schwermer for his hospitality during his visit to Eichstätt in the summer of 1993. In fact, his renewed interest on the problem was fired up by qucstions posed to him by Schwermer.

We would like to thank David Collingwood, Birgit Speh, Marko Tadić, and David Vogan for several useful communications. We would also like to thank Jean-Loup Waldspurger for communicating to us Tadić's original counterexamples to our original version of injectivity.

\section{Notation and Preliminaries}

Let $F$ be a local field of characteristic zero. When $F$ is nonarchimedean, we use $q$ to denote the number of elements in its residue field.

Throughout this paper, with the exception of Section 4, $\mathbf{G}$ denotes an arbitrary quasisplit connected reductive algebraic group over $F$. Fix a Borel subgroup $\mathbf{B}$ and write $\mathbf{B}=\mathbf{T U}$, where $\mathbf{T}$ is a maximal torus and $\mathbf{U}$ denotes the unipotent radical of $\mathbf{B}$.

Fix a parabolic subgroup $\mathbf{P}=\mathbf{M N}$ of $\mathbf{G}$ defined over $F$ with $\mathbf{N} \subset \mathbf{U}$ and $\mathbf{T} \subset \mathbf{M}$, a Levi decomposition. Let $\mathbf{A}_{0}$ be the maximal $F$-split torus of $\mathbf{T}$ and denote by $W\left(\mathbf{A}_{0}\right)$ the Weyl group of $\mathbf{A}_{0}$ in $\mathbf{G}$. Let $\widetilde{w}_{0}$ be the longest element in $W\left(\mathbf{A}_{0}\right)$ modulo that of the Weyl group of $\mathbf{A}_{0}$ in $\mathbf{M}$. Let $\psi$ be a generic character of $U=\mathbf{U}(F)$ (cf. [8,23]) and set $\psi_{M}=\psi \mid U \cap M$. Suppose $\sigma$ is an irreducible admissible $\psi_{M}$-generic representation of $M=\mathbf{M}(F)$. Changing the splitting in $\mathrm{U}$ we may assume that $\psi$ and $\widetilde{w}_{0}$ are compatible (cf. [23]).

Let $X(\mathbf{M})_{F}$ be the group of $F$-rational characters of $\mathbf{M}$. Set

$$
\mathfrak{a}^{*}=X(\mathbf{M})_{F} \otimes_{\mathbb{Z}} \mathbb{R}
$$

and

$$
\mathfrak{a}_{\mathfrak{C}}^{*}=\mathfrak{a}^{*} \otimes_{\mathbb{R}} \mathbb{C}
$$

As usual (cf. [23]), we let

$$
I(\nu, \sigma)=\operatorname{Ind}_{M N \uparrow G} \sigma \otimes q^{\left\langle\nu, H_{P}()\right\rangle} \otimes 1,
$$

where $\nu \in \mathfrak{a}_{\mathfrak{C}}^{*}$ with exp replacing $q$ if $F=\mathbb{R}$. Here $H_{P}$ is the extension of the homomorphism

$$
H_{M}: M \rightarrow \mathfrak{a}=\operatorname{Hom}\left(X(\mathbf{M})_{F}, \mathbb{R}\right)
$$

to $P$, extended trivially along $N$, where $H_{M}$ is defined by

$$
q^{\left\langle\chi, H_{P}(m)\right\rangle}=|\chi(m)|_{F}
$$

for all $\chi \in X(\mathbf{M})_{F}$.

$4^{\text {e }}$ SÉRIE - TOME $31-1998-\mathrm{N}^{\circ} 4$ 
Suppose $\mathbf{P}=\mathbf{M N}$ is maximal, $\mathbf{M} \supset \mathbf{T}$. Let $r$ be the adjoint action of ${ }^{L} M$, the $L$-group of $\mathbf{M}$, on the Lie algebra ${ }^{L} \mathfrak{n}$ of the $L$-group of $\mathbf{N}$. Then $r=\bigoplus_{i=1}^{m} r_{i}$, with $r_{i}$ 's irreducible, ordered as in [23], i.e. according to the order of eigenvalues of ${ }^{L} A$ in ${ }^{L} \mathfrak{n}$. Here ${ }^{L} A$ is the $L$-group of $\mathbf{A}$, the split component of $\mathbf{M}$. It is contained in $\mathbf{A}_{0}$. Finally, let $L\left(s, \sigma, r_{i}\right)$ be the local $L$-function attached to $\sigma$ and $r_{i}$ in [23]. (See Theorem 3.5 and Section 7 of [231.) Conjecture 7.1 of [23] plays a crucial role in the present paper and therefore for the convenience of the reader we state it here once more.

Conjecture 1.1. Assume $\sigma$ is tempered and $\operatorname{Re}(s)>0$. Then each $L\left(s, \sigma, r_{i}\right)$ is holomorphic, $1 \leq i \leq m$.

Next, assume $\mathbf{M}$ is generated by a subset $\theta$ of simple roots $\Delta$ of $\mathbf{A}_{0}$ in $\mathbf{U}$. Fix $\widetilde{w} \in W\left(\mathbf{A}_{0}\right)$ such that $\widetilde{w}(\theta) \subset \Delta$ and let $w \in G$ be a representative for $\widetilde{w}$. Let $\mathbf{N}_{\tilde{w}}=\mathbf{U} \cap u \overline{\mathbf{N}} w^{-1}$, where $\overline{\mathbf{N}}$ is unipotent subgroup opposed to $\mathbf{N}$. Given $f$ in the space of $I(\nu, \sigma)$, let

$$
A(\nu, \sigma, w) f(g)=\int_{\substack{N_{w} \\ w}} f\left(w^{-1} n g\right) d n \quad(g \in G)
$$

denote the standard intertwining operator from $I(\nu, \sigma)$ into $I(w(\nu), w(\sigma))$. It converges absolutely in some cone and extends to a meromorphic function of $\nu \in \mathfrak{a}_{\mathfrak{C}}^{*}$ (cf. [12, 16, 29]). The knowledge of its poles on all of $a_{C}^{*}$ is very important and one of the aims of the paper is to determine them in terms of $L$-functions mentioned before in certain cases.

When $\sigma$ is tempered the cone of convergence for $\nu \in \mathfrak{a}_{\mathrm{C}}^{*}$ equals to what one usually calls the positive Weyl chamber $\left(\mathfrak{a}_{\mathfrak{C}}^{*}\right)^{+}$for a. Every $\nu \in\left(\mathfrak{a}_{\mathfrak{C}}^{*}\right)^{+}$satisfies $\operatorname{Re}\left\langle\nu, H_{\alpha}\right\rangle>0$ for every $\alpha \in \Delta-\theta$ and conversely, where $H_{\alpha}$ is the standard coroot attached to $\alpha$ and $\nu$ is realized as an element of $\left(\mathfrak{a}_{0}\right)_{\mathfrak{c}}^{*}$. Here $\mathfrak{a}_{0}$ is the real Lie algebra of $\mathbf{A}_{0}$.

Suppose $\sigma$ is tempered and $\nu \in\left(\mathfrak{a}_{\mathrm{C}}^{*}\right)^{+}$. Then $I(\nu, \sigma)$ has a unique quotient $J(\nu, \sigma)$, called the Langlands quotient of $I(\nu, \sigma)$ (cf. $[4,17,28]$ ). Given an irreducible admissible representation $\pi$ of $G$, there exists a parabolic subgroup $\mathbf{P}=\mathbf{M N}, \mathbf{N} \subset \mathbf{U}, \mathbf{M} \supset \mathbf{T}$, an irreducible tempered representation $\sigma$ of $M$, and a $\nu \in\left(\mathfrak{a}_{\mathrm{C}}^{*}\right)^{+}$, such that $\pi=J(\nu, \sigma)$. Moreover, by Rodier's Theorem, $\pi$ is generic only if $\sigma$ is.

Since a part of this paper is heavily based on material in [5] and [8], we will adopt their notation in the following definitions for the convenience of the reader.

Let $\Delta$ be the set of simple roots for $\mathbf{M}_{\phi}=\mathbf{A}_{0}$ in $\mathbf{U}=\mathbf{N}_{\phi}$. Fix a subset $\theta \subset \Delta$ and let $\mathbf{P}=\mathbf{P}_{\theta}=\mathbf{M}_{\theta} \mathbf{N}_{\theta}=\mathbf{M N}, \mathbf{N}_{\theta} \subset \mathbf{N}_{\phi}$, be the corresponding standard parabolic subgroup. Let $\psi$ be a nondegenerate character of $N_{\phi}$, extending a nondegenerate character $\psi_{\theta}=\psi_{M_{\theta}}$ of $M_{\theta} \cap N_{\phi}$. Let $w_{0}$ be a representative for the element $\tilde{w}_{0}$ of the Weyl group of $\mathbf{A}_{0}$ which sends every root in $\Delta-\theta$ to a negative one, while $w_{0}(\alpha) \in \Delta$ for all $\alpha \in \theta$. Let $\mathbf{M}^{\prime}=\mathbf{M}_{\widetilde{w}_{0}(\theta)}$ and denote by $\mathbf{P}^{\prime}=\mathbf{M}^{\prime} \mathbf{N}^{\prime}, \mathbf{N}^{\prime} \subset \mathbf{N}_{\phi}$, the standard parabolic subgroup of $\mathbf{G}$ having $\mathbf{M}^{\prime}$ as its Levi factor. Assume $\psi$ and $\widetilde{w}_{0}$ are compatible (cf. [23]). This can always be achieved by changing the splitting in $\mathbf{N}_{\phi}$.

Let $(\sigma, \mathcal{H}(\sigma))$ be an irreducible admissible $\psi_{\theta}$-generic representation of $M=M_{\theta}$. Fix a Whittaker functional $\Omega_{M}$ for $\sigma$. Let $\overline{\mathbf{P}}$ be the parabolic subgroup of $\mathbf{G}$ opposed to $\mathbf{P}$. Then $\overline{\mathbf{P}}=\mathbf{M} \overline{\mathbf{N}}, \overline{\mathbf{N}}=\mathbf{N}_{-\theta}$. One can then define standard Whittaker functionals $\Omega$ and $\bar{\Omega}$ on $I(\sigma)=\operatorname{Ind}(\sigma \mid P, G)$ and $\bar{I}(\sigma)=\operatorname{Ind}(\sigma \mid \bar{P}, G)$, respectively by

$$
\langle f, \Omega\rangle=\int_{N^{\prime}} \psi^{-1}\left(n^{\prime}\right)\left\langle f\left(w_{0}^{-1} n^{\prime}\right), \Omega_{M}\right\rangle d n^{\prime}
$$


$f \in I(\sigma)$, as in $[8,25]$, and

$$
\langle\bar{f}, \bar{\Omega}\rangle=\int_{N} \psi^{-1}(n)\left\langle\bar{f}(n), \Omega_{M}\right\rangle d n,
$$

when $\bar{f} \in \bar{I}(\sigma)$. Both integrals are defined as principal values (cf. [8]).

Let

$$
T: \bar{I}(\sigma) \longrightarrow I(\sigma)
$$

be the intertwining operator

$$
T \bar{f}(g)=\int_{N} \bar{f}(n g) d n
$$

determined by the $\mathcal{H}(\sigma)$-valued functional

$$
\Lambda_{N}(\bar{f})=\int \bar{f}(n) d n
$$

Then $T \bar{f}(e)=\Lambda_{N}(\bar{f})$. Let $\gamma$ be the constant defined by Rodier's theorem, i.e.

$$
T^{*} \Omega=\gamma \bar{\Omega}
$$

Next let $\sigma^{\prime}=w_{0}(\sigma)$ and let $C_{\psi}\left(\sigma^{\prime}\right)=C_{\psi}\left(\sigma^{\prime}, w_{0}^{-1}\right)$ be the local coefficient attached to $\sigma^{\prime}, w_{0}^{-1}$, and $\psi$ (cf. [25]). More precisely it is defined by

$$
\left\langle f^{\prime}, \Omega^{\prime}\right\rangle=C_{\psi}\left(\sigma^{\prime}\right)\left\langle A\left(\sigma^{\prime}, w_{0}^{-1}\right) f^{\prime}, \Omega\right\rangle
$$

where $A\left(\sigma^{\prime}, w_{0}^{-1}\right): \operatorname{Ind}\left(\sigma^{\prime} \mid P^{\prime}, G\right) \longrightarrow \operatorname{Ind}(\sigma \mid P, G)$ is the standard intertwining operator and $\Omega^{\prime}$ is the standard Whittaker functional on $I\left(\sigma^{\prime}\right)=\operatorname{Ind}\left(\sigma^{\prime} \mid P^{\prime}, G\right)$. Finally, let $\gamma=\gamma(\sigma)$ be as in equation (1.4). We have:

LEMMA 1.2. $-\gamma=C_{\psi}\left(\sigma^{\prime}, w_{0}^{-1}\right)^{-1}$.

Proof. - Let $\bar{I}(\sigma)=\operatorname{Ind}(\sigma \mid \bar{P}, G)$ and let

$$
T: \bar{I}(\sigma) \longrightarrow I(\sigma)
$$

be the intertwining operator (2.3). If

$$
L_{w_{0}}: I\left(\sigma^{\prime}\right) \longrightarrow \bar{I}(\sigma)
$$

is defined by $L_{w_{0}} f^{\prime}(g)=f^{\prime}\left(w_{0} g\right)$, then

$$
T \cdot L_{w_{0}}=A\left(\sigma^{\prime}, w_{0}^{-1}\right) .
$$

By definition

$$
\left\langle\bar{f}, T^{*} \Omega\right\rangle=\gamma\langle\bar{f}, \bar{\Omega}\rangle
$$

where $\bar{f}=L_{w_{0}} f^{\prime}, f^{\prime} \in I\left(\sigma^{\prime}\right)$. Then

$$
\left\langle L_{w_{0}} f^{\prime}, \bar{\Omega}\right\rangle=\left\langle f^{\prime}, \Omega^{\prime}\right\rangle
$$

$4^{\mathrm{e}}$ SÉRIE - TOME $31-1998-\mathrm{N}^{\circ} 4$ 
with $\Omega^{\prime}$ as above. Using (1.2.1) and (1.2.3), (1.2.2) implies

$$
\left\langle A\left(\sigma^{\prime}, w_{0}^{-1}\right) f^{\prime}, \Omega\right\rangle=\gamma\left\langle f^{\prime}, \Omega^{\prime}\right\rangle
$$

from which the lemma is immediate.

If $\sigma$ is a $\psi$-generic irreducible tempered representation of $M$ and $\nu \in \mathfrak{a}_{\mathfrak{C}}^{*}$, we use $C_{\psi}(\nu, \sigma)$ to simply denote the local coefficient [25] attached to $\nu, \sigma$, and $w_{0}$, i.e. the complex function defined by

$$
\langle f, \Omega\rangle=C_{\psi}(\nu, \sigma)\left\langle A\left(\nu, \sigma, w_{0}\right) f, \Omega^{\prime}\right\rangle
$$

where

$$
A\left(\nu, \sigma, w_{0}\right): I(\nu, \sigma) \rightarrow I\left(w_{0}(\nu), w_{0}(\sigma)\right)
$$

is the standard intertwining operator defined earlier.

\section{Irreducibility of standard modules}

Let $\pi$ be an irreducible admissible generic representation of $G$. By Langlands classification ([4, 17, 28]) there exists a standard parabolic subgroup $\mathbf{P}=\mathbf{M N}$ of $\mathbf{G}$ with split component $\mathbf{A}$, an irreducible generic tempered representation $\sigma$ of $M$ and a $\nu$ in the positive Weyl chamber of $\mathfrak{a}_{\complement}^{*}$ such that $\pi=J(\nu, \sigma)$, the (unique) Langlands quotient of $I(\nu, \sigma)$. When $F=\mathbb{R}$, in [37], Vogan proved:

Theorem 2.1 (Vogan). - Assume $F=\mathbb{R}$ and $\pi=J(\nu, \sigma)$ is generic. Then $J(\nu, \sigma)=$ $I(\nu, \sigma)$. In particular $I(\nu, \sigma)$ is irreducible.

Now suppose $F$ is $p$-adic. In this section we extend Theorem 2.1 to $p$-adic fields when $\sigma$ is supercuspidal. More precisely, we prove:

THEOREM 2.2. - Let $\sigma$ be an irreducible generic unitary supercuspidal representation of M. Assume $J(\nu, \sigma)$ is generic. Then $I(\nu, \sigma)=J(\nu, \sigma)$, i.e., $I(\nu, \sigma)$ is irreducible.

Proof. - Assume $\sigma$ is $\psi_{\theta}$-generic and let $\psi$ be an extension of $\psi_{\theta}$ (cf. Section 1). Let $C_{\psi}\left(w_{o}(\nu), w_{0}(\sigma)\right)$ be the corresponding local coefficient, i.e. the complex number defined by

$$
\left\langle f^{\prime}, \Omega^{\prime}\right\rangle=C_{\psi}\left(w_{0}(\nu), w_{0}(\sigma)\right)\left\langle A\left(w_{0}(\nu), w_{0}(\sigma), w_{0}^{-1}\right) f^{\prime}, \Omega\right\rangle
$$

where $\Omega^{\prime}$ is the standard Whittaker functional on $I\left(w_{0}(\nu), w_{0}(\sigma)\right)$. By Proposition 7.3 of [23], Conjecture 1.1 is true and therefore the denominator of $C_{\psi}\left(w_{0}(\nu), w_{0}(\sigma)\right)$ is a product of the form

$$
\prod_{j} L\left(1+s_{j}, w_{0}(\sigma), \widetilde{r}_{j}\right)^{1}
$$

where $L$-functions are as in [23] with $\operatorname{Re}\left(s_{j}\right)>0$, since $\nu$ is in the positive Weyl chamber (Theorem 3.5 of [23], equation (3.11)). Again by Proposition 7.3 of [23], this product is non-zero since these $L$-functions are holomorphic if $\operatorname{Re}\left(s_{j}\right)>0$ (in fact if $\operatorname{Re}\left(s_{j}\right) \neq-1$, 
$\sigma$ being supercuspidal). In conclusion $C_{\psi}\left(w_{0}(\nu), w_{0}(\sigma)\right)$ is well defined whenever $\nu$ is in the positive Weyl chamber.

On the other hand, by Theorem 5.4.2.1 of [29], the operator $A\left(w_{0}(\nu), w_{0}(\sigma), w_{0}^{-1}\right)$ is holomorphic as long as $\sigma$ is unitary supercuspidal and $\nu$ is regular, and in particular if $\nu$ is in the positive Weyl chamber. It now follows that the normalized operator

$$
C_{\psi}\left(w_{0}(\nu), w_{0}(\sigma)\right) A\left(w_{0}(\nu), w_{0}(\sigma), w_{0}^{-1}\right)
$$

is well defined on all of $I\left(w_{0}(\nu), w_{0}(\sigma)\right)$ if $I(\nu, \sigma)$ is standard.

Choose $f^{\prime}$ in the space of $I\left(w_{0}(\nu), w_{0}(\sigma)\right)$ such that $\left\langle f^{\prime}, \Omega^{\prime}\right\rangle \neq 0$. Then by equation (2.2.1), the image of $f^{\prime}$ under (2.2.2) is $\psi$-generic.

Suppose $J(\nu, \sigma)$ is $\psi$-generic, but $I(\nu, \sigma)$ is reducible. The image of $I\left(w_{0}(\nu), w_{0}(\sigma)\right)$ under (2.2.2) being $\psi$-generic, will have an irreducible $\psi$-generic subquotient which is inequivalent to $J(\nu, \sigma)$ by uniqueness of Langlands quotient. This contradicts Rodier's Theorem and proves the Theorem.

Remark 2.3. - The standard modules which are built by means of minimal parabolic subgroups are clcarly among spccial cases covcred by Thcorem 2.2, since quasicharacters of $A_{\phi}$, the $F$-points of the split component of $\mathbf{M}_{\phi}$ are supercuspidal. When $\sigma$ is an unramified character of $A_{\phi}$, the theorem was proved in [2], [20], and [22], earlier, each using a very different method.

Corollary 2.4. - Let $\gamma$ be as in equation (1.4) and assume $I(\sigma)=\operatorname{Ind}(\sigma \mid P, G)$ is standard. Then $\gamma \neq 0$.

Proof. - This follows from Lemma 1.2 and the fact that $C_{\psi}\left(\sigma^{\prime}\right)=C_{\psi}\left(w_{0}(\sigma)\right)$ is well defined which was observed in the proof of Theorem 2.2, if $\sigma$ is in the positive Weyl chamber.

\section{Generalized injectivity}

In this section we will address a property of standard modules which implies Vogan's theorem for them and is therefore rather stronger. It simply requires the generic constituents of a standard module to become subrepresentations.

DEFINITION 3.1. - A standard module is said to satisfy injectivity if all its irreducible subrepresentations are generic.

Remark. - When the inducing data is supercuspidal, we will show (Theorem 3.4) that the corresponding standard module satisfies injectivity. We initially believed that every standard module for a $p$-adic group satisfies injectivity. In fact, as opposed to the case of real groups for which already the rank one quasisplit group $U(2,1)$ has standard modules which do not satisfy injectivity [4], standard modules defined by supercuspidal data for any $p$-adic quasisplit group do. But certain recent examples of Tadic for $G S p_{2 n}, n \geq 4$, has convinced us to contrary in general. In fact, Tadic has a class of counterexamples for classical groups which are now included in his new version of [34]. What follows are some low rank examples which were communicated to us by him.

The lowest rank examples of Tadić are for $G S p_{8}(F)$ and $S O_{7}(F)$. In hoth groups $T=A_{0}$ acts transitively on the set of generic characters of $U$. Let $\sigma_{0}$ denote the Steinberg

$4^{\mathrm{e}}$ SÉRIE - TOME $31-1998-\mathrm{N}^{\circ} 4$ 
representation of $G L_{n}(F), n=3$ or 4 , a non-supercuspidal discrete series representation. If $G=G S p_{8}(F)$, let $\sigma=\sigma_{0} \otimes|\operatorname{det}()|^{\frac{1}{2}} \otimes 1$, a representation of $G L_{4}(F) \times G L_{1}(F)$, the Siegel Levi subgroup of $C S p_{8}(F)$. On the other hand for $G=S O_{7}(F)$, let $\sigma=\sigma_{0} \otimes \mid \operatorname{det}()^{\frac{1}{2}}$ denote one for $G L_{3}(F)$, the Siegel Levi subgroup of $S O_{7}(F)$. In either case, using Jacquet modules, Tadić shows that the standard module $I(\sigma)$ has two (non-isomorphic) irreducible subrepresentations. By the above remark one of them must be degenerate.

We therefore nced the following definition.

Definition 3.2. - A standard module is said to satisfy generalized injectivity if all its generic constituents appear as subrepresentations.

CONJFCTURF 3.3. - Every standard module satisfies generalized injectivity.

Remark. - The conjecture seems to be valid for real groups although no proof for it has been published (private communications with Vogan). In this section we will prove the conjecture when $F$ is $p$-adic and the inducing data is supercuspidal.

THEOREM 3.4. - Let $F$ be a p-adic field of characteristic zero and let $\mathbf{G}$ be a quasisplit group over $F$. Let $I(\nu, \sigma)$ be a standard module for $G$. Assume $\sigma$ is generic and supercuspidal. Then the unique irreducible subrepresentation of $I(\nu, \sigma)$ is generic, i.e., injectivity is valid for supercuspidal inducing data. In other words $I(\nu, \sigma)$ injects into $\operatorname{Ind}_{U \uparrow G} \psi$ for every $\psi$ extending $\psi_{\theta}$, the character with respect to which $\sigma$ is generic.

We use notation as in Section 1. More precisely, let

$$
\sigma_{\nu}=\sigma \otimes q^{\left\langle\nu, H_{M}()\right\rangle} .
$$

Then we set $I\left(\sigma_{\nu}\right)=I(\sigma, \nu)$ and use $I$ and $V$ to denote $I\left(\sigma_{\nu}\right)$ and the space $V\left(\sigma_{\nu}\right)=V(\nu, \sigma)$ of $I(\nu, \sigma)$.

Since $\sigma_{\nu}$ is supercuspidal and regular, Proposition 6.4.1 of [5] implies that $\sigma_{\nu} \delta^{1 / 2}$ appears in $V_{N}=V_{N_{\theta}}$, the Jacquet module of $V$ with respect to $N$, with multiplicity one. Consequently by Frobenius reciprocity $V$ has a unique irreducible subspace. Theorem 3.4 then requires this subrepresentation to be generic and in particular it will be generic with respect to every extension $\psi$ of $\psi_{\theta}$. We start with two lemmas. Again the notation is as in Section 1.

Lemma 3.5. - Suppose $\bar{f} \in \operatorname{Ind}(\sigma \mid \bar{P}, G)$ has support in $\bar{P} N$. Then there exists $\varepsilon=\varepsilon(\bar{f})>0$ such that

$$
\langle\bar{I}(a) \bar{f}, \bar{\Omega}\rangle=\delta_{P}^{1 / 2}(a) \sigma_{\nu}(a)\left\langle\Lambda_{N}(\bar{f}), \Omega_{M}\right\rangle
$$

for every $a \in A_{\theta}$ with $|\alpha(a)|<\varepsilon, \alpha \in \Delta-\theta$. Here $\mathbf{A}_{\theta}$ is the split component of $\mathbf{M}_{\theta}, \bar{I}=\bar{I}\left(\sigma_{\nu}\right)$, and $\delta_{P}$ is the modulus character of $P$.

Proof. - Assume $\bar{f}$ is supported in $\bar{P} \omega$. Then

$$
\begin{aligned}
\langle\bar{I}(a) \bar{f}, \bar{\Omega}\rangle & =\int_{N} \psi^{-1}(n)\left\langle\bar{f}(n a), \Omega_{M}\right\rangle d n \\
& =\int_{N} \psi^{-1}(n) \delta^{-1 / 2}(a) \sigma_{\nu}(a)\left\langle\bar{f}\left(a^{-1} n a\right), \Omega_{M}\right\rangle d n \\
& =\int_{N} \psi^{-1}\left(a n a^{-1}\right) \delta^{1 / 2}(a) \sigma_{\nu}(a)\left\langle\bar{f}(n), \Omega_{M}\right\rangle d n .
\end{aligned}
$$


Now choose $\varepsilon$ small enough so that $\psi \mid a \omega a^{-1} \equiv 1$, concluding the lemma.

Let $V$ be the space of $I\left(\sigma_{\nu}\right)$ and denote by $V_{N_{\theta}}$ its Jacquet module with respect to $N=N_{\theta}$, where $\theta$ is the subset of $\Delta$ generating $\mathbf{M}=\mathbf{M}_{\theta}$. The space of Whittaker functionals on $V_{N_{\theta}}$ is the dual of $\left(V_{N_{\theta}}\right)_{\psi_{\theta}, M_{\theta} \cap N_{\phi}}$ (notation as in [8]).

With notation as in [5] and [8], consider the diagram

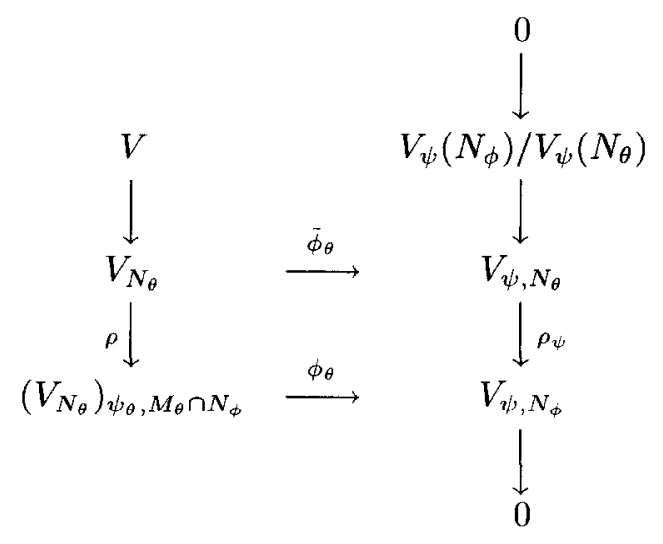

in which $\widetilde{\phi}_{\theta}$ is defined by means of canonical liftings (cf. Section 4 of [5]), exactly as in the minimal case in [8]. It can be easily shown that $\operatorname{ker}(\rho) \subset \operatorname{ker}\left(\rho_{\psi} \cdot \widetilde{\phi}_{\theta}\right)$, leading to the well defined map $\phi_{\theta}$ of Proposition 6.4 of [8]. Consequently $\Omega \cdot \phi_{\theta}$ defines a functional on $\left(V_{N_{\theta}}\right)_{\psi_{\theta}, M_{\theta} \cap N_{\phi}}$ and is therefore a Whittaker functional for $V_{N_{\theta}}$. Here $\Omega=\lambda_{\psi}(\nu, \sigma)$ is the canonical Whittaker functional for $I(\nu, \sigma)$ defined in Section 1. We now restate Proposition 6.4 of [8] in our case as follows.

LEMMA 3.6. - Given $f$ in the space $V$ of $I(\nu, \sigma)$, there exists $\varepsilon>0$ such that

$$
\langle I(a) f, \Omega\rangle=\Omega \cdot \phi_{\theta}\left(I_{N_{\theta}}(a) \tilde{f}\right)
$$

for all $a \in A_{\theta}$ with $|\alpha(a)|<\varepsilon, \forall \alpha \in \Delta-\theta$. Here $\tilde{f}$ is the image of $f$ in $V_{N_{\theta}}$.

Proof of Theorem 3.4. - By Proposition 6.4.1 of [5]

$$
I_{N}=I_{N_{\theta}}=\bigoplus_{w \in W(\mathbf{A})}\left(w \sigma_{\nu}\right) \delta^{1 / 2}
$$

where the sum is over the Weyl group of $\mathbf{A}$ in $\mathbf{G}$. Since $\sigma_{\nu}$ is regular, each irreducible subrepresentation appears with multiplicity one in (3.4.1). Given $f \in I\left(\sigma_{\nu}\right)$, write

$$
\tilde{f}=\bigoplus_{w} \tilde{f}_{w}
$$

where $\tilde{f}_{w}$ is in $\left(w \sigma_{\nu}\right) \delta^{1 / 2}$. Here $\tilde{f}$ is the image of $f$ in $I_{N}$. By Frobenius reciprocity

$$
I_{N_{\theta}}(m) \widetilde{f_{1}}=\sigma_{\nu}(m) \delta^{1 / 2}(m) f(e) \quad\left(m \in M_{\theta}\right) .
$$

For cach $w$, let $\lambda_{w}$ be a Whittaker functional for $\left(w \sigma_{\nu}\right) \delta^{1 / 2}$. We will take $\lambda_{1}=\Omega_{M}$. Write

$$
\Omega \cdot \phi_{\theta}=\sum_{w} c_{w} \lambda_{w}
$$

$4^{\text {e }}$ SÉRIE - TOME $31-1998-\mathrm{N}^{\circ} 4$ 
using the fact that $\Omega \cdot \phi_{\theta}$ is a Whittaker functional for $V_{N_{\theta}}$. By Lemma 3.6 there exists $\varepsilon=\varepsilon(f)>0$ such that

$$
\langle I(a) f, \Omega\rangle=\Omega \cdot \phi_{\theta}\left(I_{N_{\theta}}(a) \tilde{f}\right)=\sum_{w} c_{w}(w \sigma)(a) \delta^{1 / 2}(a)\left\langle\tilde{f}_{w}, \lambda_{w}\right\rangle
$$

for all $a \in \Lambda_{\theta}=A$ with $|\alpha(a)|<\varepsilon, \forall \alpha \in \Delta-\theta$.

Next, choose $\bar{f} \in \bar{I}\left(\sigma_{\nu}\right)$ with support in $\bar{P} N$ such that $T \bar{f}(e) \neq 0$. Such functions exist by Lemma 4.2 of [27]. By Lemma 3.5 and equation (1.4), there exists $\varepsilon=\varepsilon(\bar{f})>0$ such that

$$
\langle I(a)(T \bar{f}), \Omega\rangle=\gamma \sigma_{\nu} \delta^{1 / 2}(a)\left\langle(T \bar{f})(e), \Omega_{M}\right\rangle
$$

for $a \in A_{\theta}$ with $|\alpha(a)|<\varepsilon, \forall \alpha \in \Delta-\theta$.

Since $\sigma_{\nu}$ is regular, Theorem 5.4.2.1 of [29] implies that $T \cdot L_{w_{0}}$ is well defined on all of $I\left(w_{0}(\nu), w_{0}(\sigma)\right)$ where

$$
T: \bar{I}\left(\sigma_{\nu}\right) \rightarrow I\left(\sigma_{\nu}\right)
$$

is defined by (1.3) and

$$
L_{w_{0}}: I\left(\sigma_{\nu}^{\prime}\right)=I\left(w_{0}(\nu), w_{0}(\sigma)\right) \rightarrow \bar{I}\left(\sigma_{\nu}\right)
$$

is as in Lemma 1.2. Consequently $f=T \bar{f}$ is well defined and belongs to $I\left(\sigma_{\nu}\right)$. Comparing (3.4.3) with (3.4.2) and using the regularity of $\sigma_{\nu}$, one has $c_{1}=\gamma$ which is non-zero by Corollary 2.4 . We can therefore write

$$
\langle I(a) f, \Omega\rangle=\gamma \sigma_{\nu} \delta^{1 / 2}(a)\left\langle f(e), \Omega_{M}\right\rangle+\sum_{w \neq 1} c_{w} \cdot\left(w \sigma_{\nu}\right)(a) \delta^{1 / 2}(a)\left\langle\tilde{f}_{w}, \lambda_{w}\right\rangle
$$

Since $\nu$ is in the positive Weyl chamber, the term

$$
\gamma \sigma_{\nu} \delta^{1 / 2}(a)\left\langle f(e), \Omega_{M}\right\rangle
$$

is now a leading term as $|\alpha(a)| \rightarrow 0, a \in A_{\theta}, \forall \alpha \in \Delta-\theta$, if $\left\langle f(e), \Omega_{M}\right\rangle \neq 0$.

Suppose $0 \neq f \in V$ is such that $\langle I(g) f, \Omega\rangle=0$ for all $g \in G$. Then $W_{f}=0$, where $W_{f}(g)=\langle I(g) f, \Omega\rangle$, i.e., $0 \neq f$ lies in the kernel of $f \mapsto W_{f}$ into $\operatorname{Ind}_{U \dagger G} \psi$ or injectivity fails.

We may assume $\left\langle f(e), \Omega_{M}\right\rangle \neq 0$. Then for $a \in A_{\theta}$ with $|\alpha(a)|<\varepsilon=\varepsilon(f), \forall \alpha \in \Delta-\theta$,

$$
0=\langle I(a) f, \Omega\rangle=\gamma \sigma_{\nu} \delta^{1 / 2}(a)\left\langle f(e), \Omega_{M}\right\rangle+\cdots
$$

with $\gamma \sigma_{\nu} \delta^{1 / 2}(a)\left\langle f(e), \Omega_{M}\right\rangle$, a non-zero leading term as $|\alpha(a)| \mapsto 0, \forall \alpha \in \Delta-\theta$. This is a contradiction. 


\section{Proof of Conjecture 1.1 for Groups of Classical Type}

Throughout this paper, a classical group is a connected algebraic group, fixing a nondegenerate bilinear form of either symmetric, alternating, or Hermitian type. The group $\mathbf{G}$ will be called of classical type if there exists a product of classical groups whose derived group is a covering, as an algebraic group, of the derived group of $\mathbf{G}$. We will further assume that $\mathbf{G}$ is quasisplit. The purpose of this section is to prove Conjecture 1.1 when $\mathbf{G}$ is of classical type.

Let $\mathbf{G}$ be a quasi-split classical group over a $p$-adic field $F$ of characteristic zero. Let $\sigma_{2}^{\vee}$ be a discrete series representation of $G=\mathbf{G}(F)$. Choose a Levi subgroup

$$
\mathbf{M}=G L_{r_{1}} \times \ldots \times G L_{r_{n}} \times \mathbf{G}^{0}
$$

of $\mathbf{G}$, with $\mathbf{G}^{0}$ classical. When $\mathbf{G}$ is the unitary group defined by a quadratic extension $E / F, G L_{r_{i}}$ must be replaced by $\operatorname{Res}_{E / F} G L_{r_{i}}$. The reader must be warned that $\sigma_{2}^{\vee}$ is a pure symbol and is neither the dual nor the contragredient of a representation $\sigma_{2}$. Choose an irreducible supercuspidal representation $\sigma_{2}=\rho_{1} \otimes \ldots \otimes \rho_{n} \otimes \tau$ of $M=\mathbf{M}(F)$ such that

$$
\sigma_{2}^{\vee} \subset \operatorname{Ind}_{M N}^{G} \sigma_{2} \otimes \mathbf{1}
$$

Next, let $\sigma_{0}$ be an irreducible unitary supercuspidal representation of $G L_{t}(F)$. Assume $\tau$ is generic. Let $\sigma_{1}^{\vee}$ be a discrete series representation of $G L_{u}(F)$ with $t \mid u$, defined by means of $\sigma_{0}$ as in [3, 41]. (See below.)

We are interested in the Rankin-Selberg product factors for $\sigma_{1}^{\vee} \times \sigma_{2}^{\vee}$. More precisely we want to study

$$
L\left(s, \sigma_{1}^{\vee} \times \sigma_{2}^{\vee}\right) \quad(s \in \mathbb{C})
$$

as defined by the $\gamma$-function $\gamma\left(s, \sigma_{1}^{\vee} \times \sigma_{2}^{\vee}, \psi_{F}\right)$ of Theorem 3.5 of [23]. (See Section 7.) Here $\gamma\left(s, \sigma_{1}^{\vee} \times \sigma_{2}^{\vee}, \psi_{F}\right)$ is $\gamma\left(s, \sigma_{1}^{\vee} \otimes \sigma_{2}^{\vee}, r_{1}, \psi_{F}\right)$ of cases $B_{n}, C_{n}, D_{n}-1,{ }^{2} A_{n}-4$, and ${ }^{2} D_{n}-1$, chosen according to $G$. For the sake of simplicity, from now on, we drop the dependence on $\psi_{F}$ from $\gamma\left(s, \sigma_{1}^{\vee} \times \sigma_{2}^{\vee}, \psi_{F}\right)$. As in Corollary 5.6 of [26] we have:

$$
\gamma\left(s, \sigma_{0} \times \sigma_{2}^{\vee}\right)=\gamma\left(s, \sigma_{0} \times \tau\right) \prod_{j=1}^{n} \gamma\left(s, \sigma_{0} \times \rho_{j}\right) \gamma\left(s, \sigma_{0} \times \tilde{\rho}_{j}\right)
$$

for the pair $\left(\sigma_{0}, \sigma_{2}^{\vee}\right)$.

For each $j, 1 \leq j \leq n$, choose an irreducible unitary supercuspidal representation $\rho_{0, j}$ and a real number $\nu_{j}$ such that $\rho_{j}=\rho_{0, j} \otimes|\operatorname{det}(\quad)|^{\nu_{j}}$ :

Let $\rho=\rho_{1} \otimes \ldots \otimes \rho_{n}$ and set

$$
\eta\left(s, \sigma_{0}, \tau, \rho\right)=L\left(s, \sigma_{0} \times \tau\right) \prod_{j=1}^{n} L\left(s+\nu_{j}, \sigma_{0} \times \rho_{0, j}\right) L\left(s-\nu_{j}, \sigma_{0} \times \ddot{\rho}_{0, j}\right) .
$$

The reader must realize that when we are in the unitary case $A_{n}-4$ of $[23], \tilde{\rho}_{0, j}$ must be replaced by $\tilde{\bar{\rho}}_{0, j}$ throughout, where $\bar{\rho}_{0, j}$ denotes the Galois conjugate of $\rho_{0, j}$ under the non-trivial element of the Galois group of the defining quadratic extension.

$4^{\mathrm{e}}$ SÉRIE - TOME $31-1998-\mathrm{N}^{\circ} 4$ 
If $A(s)$ and $B(s)$ are two rational functions in $q^{-s}$, we use $A(s) \sim B(s)$ if they arc equal up to a monomial in $q^{-s}$. Then from (4.1) and (4.2) we have

$$
\gamma\left(s, \sigma_{0} \times \sigma_{2}^{\vee}\right) \sim \eta\left(s-1, \sigma_{0}, \tau, \rho\right) / \eta\left(s, \sigma_{0}, \tau, \rho\right) .
$$

Now suppose $\sigma_{1}^{\vee}$ is the unique discrete series constituent of

$$
\operatorname{Ind}_{\Pi G L_{t}(F) \cdot N}^{G L_{u}(F)}\left(\sigma_{1} \otimes \ldots \otimes \sigma_{b}\right) \otimes \mathbf{1},
$$

where $\Pi G L_{t}(F)$ has $b$ factors with $b=u / t$ and $\sigma_{i}=\sigma_{0} \otimes|\operatorname{det}(\quad)|^{(b+1) / 2-i}, 1 \leq i \leq b$. (See $[3,41]$.)

Then

$$
\begin{aligned}
\gamma\left(s, \sigma_{1}^{\vee} \times \sigma_{2}^{\vee}\right) & =\prod_{i=1}^{b} \gamma\left(s+(b+1) / 2-i, \sigma_{0} \times \sigma_{2}^{\vee}\right) \\
& =\prod_{i=1}^{b} \gamma\left(s, \sigma_{i} \times \sigma_{2}^{\vee}\right) \\
& \sim \prod_{i=1}^{b} \eta\left(s+(b+1) / 2-i-1, \sigma_{0}, \tau, \rho\right) / \eta\left(s+(b+1) / 2-i, \sigma_{0}, \tau, \rho\right) .
\end{aligned}
$$

Define $L\left(s, \sigma_{1}^{\vee} \times \sigma_{2}^{\vee}\right)^{-1}$ as the numerator of $\gamma\left(s, \sigma_{1}^{\vee} \times \sigma_{2}^{\vee}\right)$ as in [23]. We shall prove:

THEOREM 4.1. - The L-function $L\left(s, \sigma_{1}^{\vee} \times \sigma_{2}^{\vee}\right)$ is holomorphic for $\operatorname{Re}(s)>0$, i.e. Conjecture 1.1 is valid.

The following corollary is then a consequence of part 2 of Theorem 3.5 of [23] and Theorem 4.1 here since the local coefficients for groups of classical type are just a product of those for classical groups. Observe that it states a result on the holomorphy of local coefficients which is usually deep.

COROLlary 4.2. - Let $\mathbf{G}$ be of classical type. Then the corresponding local coefficient for any parabolic subgroup and any generic tempered representation of its Levi factor is holomorphic for $\nu \in \widetilde{w}_{0}\left(\left(\mathfrak{a}_{\mathfrak{C}}^{*}\right)^{+}\right)$.

By Lemma 4.1 of [34], representations $\rho_{0, j}$ are all self-contragredient. In fact, although in [34] this is only proved for symplectic and odd special orthogonal groups, we have been assured by Tadić that similar results can be proved for other classical groups. Similar remark applies to Lemma 4.6 of [34]. (See Remark 4.21 below.) Lemma 4.1 has also been verified independently in a work in progress by $\mathrm{Y}$. Zhang, using results of Harish-Chandra and Silberger on special orbits (cf. [28]).

The $L$-functions in the product

$$
\prod_{j=1}^{n} L\left(s+\nu_{j}, \sigma_{0} \times \rho_{0, j}\right) L\left(s-\nu_{j}, \sigma_{0} \times \rho_{0, j}\right)
$$

are non-trivial only if $\sigma_{0} \otimes|\operatorname{det}(\quad)|^{s_{0, j}} \cong \rho_{0, j}$ for some $s_{0, j} \in i \mathbb{R}$. We therefore may assume, by shifting $s$ by $s_{0, j} \in i \mathbb{R}$, that $\sigma_{0}$ is self-dual and $\rho_{0, j} \cong \sigma_{0}$. We shall therefore need to study products of type

$$
L\left(s, \sigma_{0} \times \tau\right) \prod_{j=1}^{n} L\left(s+\nu_{j}, \sigma_{0} \times \sigma_{0}\right) L\left(s-\nu_{j}, \sigma_{0} \times \sigma_{0}\right),
$$


where $\sigma_{0} \cong \tilde{\sigma}_{0}$ and $\nu_{j} \in \mathbb{R}$. Observe that we may need to study these products with different values of $s$. But $\operatorname{Re}(s)$ will be the same for all such products.

Given $t \in \mathbb{C}$, set

$$
L(t)=L\left(t, \sigma_{0} \times \sigma_{0}\right)
$$

whose dependence on $\sigma_{0}$ is understood.

Definition 4.3. - By a chain based on $\sigma_{0}$ or a $\sigma_{0}$-chain (simply a chain if $\sigma_{0}$ is fixed), we mean a sequence of representations

$$
\sigma_{j}=\sigma_{0} \otimes|\operatorname{det}()|^{\nu_{j}} \quad\left(\nu_{j} \in \mathbb{R}\right)
$$

for which $\nu_{j}-\nu_{j-1}=1$ for all possible $j$. If $\sigma_{0}$ is understood to be fixed, we use $\left\{\nu_{j}\right\}_{j=1}^{n}$ to denote a chain based on $\sigma_{0}$. This is what is called a segment in [34] and [41].

Using results from [23], we shall first prove that one only needs a union of ccrtain special types of chains to obtain all the induced representations which have discrete series subrepresentation. Our main tool is the following theorem whose proof is an application of Proposition 7.2.b of [23]. One observes that by Proposition 7.3 of [23] the assumption on $L\left(s, \sigma, r_{2}\right)=L\left(s, \sigma_{0}, r_{2}\right)$ is in fact satisfied.

THEOREM 4.4. - Define $L\left(s, \sigma_{0} \times \sigma_{2}^{\vee}\right)$ as the inverse of the numerator of $\gamma\left(s, \sigma_{0} \times \sigma_{2}^{\vee}\right)$, where $\sigma_{2}^{\vee}$ is in the discrete series and $\sigma_{0}$ is irreducible unitary supercuspidal. Then $L\left(s, \sigma_{0} \times \sigma_{2}^{\vee}\right)$ is holomorphic for $\operatorname{Re}(s)>0$.

We start with the following lemma.

Lemma 4.5. - Fix complex numbers $\mu$ and $\nu$ and let $\sigma_{\mu}=\sigma_{0} \otimes|\operatorname{det}(\quad)|^{\mu}$ and $\sigma_{\nu}=\sigma_{0} \otimes|\operatorname{det}()|^{\nu}$. Then $L\left(s, \sigma_{0} \times \sigma_{\mu}\right)^{-1}$ and $L\left(s, \sigma_{0} \times \sigma_{\nu}\right)^{-1}$ have a factor in common as polynomials in $q^{-s}$ if and only if $\sigma_{\mu} \cong \sigma_{\nu}$. In this case $L\left(s, \sigma_{0} \times \sigma_{\mu}\right)=L\left(s, \sigma_{0} \times \sigma_{\nu}\right)$.

Proof. - The $L$-function $L\left(s, \sigma_{0} \times \sigma_{0}\right)=\prod_{\eta}\left(1-\eta(\varpi) q^{-s}\right)^{-1}$, where the product is over the group of all the unramified characters which fix $\sigma_{0}$. Then

$$
\begin{aligned}
L\left(s, \sigma_{0} \times \sigma_{\mu}\right) & =L\left(s+\mu, \sigma_{0} \times \sigma_{0}\right) \\
& =\prod_{\eta}\left(1-\eta \eta_{0}(\varpi) q^{-s}\right)^{-1},
\end{aligned}
$$

where $\eta_{0}=||^{\mu}$, while

$$
L\left(s, \sigma_{0} \times \sigma_{\nu}\right)=\prod_{\eta}\left(1-\eta \eta_{0}^{\prime}(\varpi) q^{-s}\right)^{-1}
$$

where $\eta_{0}^{\prime}=||^{\nu}$. If they have any factors in common, then $\eta_{0} / \eta_{0}^{\prime}$ will have to belong to the group of unramified stabilizers of $\sigma$ and conversely.

Let $\left\{\nu_{j}\right\}_{j=1}^{n}$ be a chain based on a self-dual irreducible supercuspidal representation $\sigma_{0}$ of $G L_{t}(F)$. Let $\sigma_{2}^{\vee}$ be a discrete series representation of $G$. Assume there exists a Levi subgroup $\mathbf{M}$ of $\mathbf{G}$ which is a direct product of an $n$-product of $G L_{t}$ with a Levi subgroup $\mathbf{M}^{\prime}$ of a smaller rank similar classical group and an irreducible supercuspidal representation $\sigma_{2}^{\prime}$ of $M^{\prime}=\mathbf{M}^{\prime}(F)$ such that $\sigma_{2}^{\vee}$ can be embedded as a subrepresentation of

$4^{\text {e }}$ SÉRIE - TOME $31-1998-\mathrm{N}^{\circ} 4$ 
the representation of $G$ induced from $\left(\prod_{j=1}^{n} \sigma_{0} \otimes|\operatorname{det}(\quad)|^{\nu_{j}}\right) \otimes \sigma_{2}^{\prime}$ of $\prod G L_{t}(F) \times M^{\prime}$. We then say $\sigma_{2}^{\vee}$ is partially supported by the chain $\left\{\nu_{j}\right\}_{j=1}^{n}$.

Definition 4.6. - Let $\left\{\nu_{j}\right\}_{j=1}^{n}$ be a $\sigma_{0}$-chain. We shall call

$$
\begin{aligned}
\gamma\left(s ; \nu_{1}, \ldots, \nu_{n}\right) & =\prod_{j=1}^{n} \frac{L\left(s-\nu_{j}-1\right) L\left(s+\nu_{j}-1\right)}{L\left(s-\nu_{j}\right) L\left(s+\nu_{j}\right)} \\
& =\frac{L\left(s-\nu_{n}-1\right) L\left(s+\nu_{1}-1\right)}{L\left(s-\nu_{1}\right) L\left(s+\nu_{n}\right)}
\end{aligned}
$$

the $\gamma$-function of the chain $\left\{\nu_{j}\right\}_{j=1}^{n}$.

Definition 4.7. - Two $\sigma_{0}$-chains are called $\gamma$-equivalent, if they have equal $\gamma$-functions.

LEMMA 4.8. - Every non-positive chain is $\gamma$-equivalent to a non-negative one.

Proof. - Use $\gamma\left(s ; \nu_{1}, \ldots, \nu_{n}\right)=\gamma\left(s ;-\nu_{n}, \ldots,-\nu_{1}\right)$.

LEMMA 4.9. - If there exists only one chain $\left\{\nu_{j}\right\}_{j=1}^{n}$ with $\nu_{1} \geq 0$ giving the $\sigma_{0}$-support of $\sigma_{2}^{\vee}$, then either $\nu_{1}=0, \nu_{1}=1 / 2$, or $\nu_{1}=1$.

Proof. - If $\nu_{1}>0$, then by Theorem 4.4, $L\left(s-\nu_{1}\right)$ must not appear in $\gamma\left(s, \sigma_{0} \times \sigma_{2}^{\vee}\right)$. By Lemma 4.5 we may assume that, either $L\left(s-\nu_{1}\right)^{-1}$ cancels $L\left(s+\nu_{1}-1\right)^{-1}$, or it divides $L\left(s-1, \sigma_{0} \times \tau\right)^{-1}$. In the first case, again by Lemma 4.5 , we may assume $\nu_{1}-1=-\nu_{1}$, since their difference fixes $\sigma_{0}$. The other case could only possibly happen if $\nu_{1}=1$. One needs only to observe that $L\left(s, \sigma_{0} \times \tau\right)$ has no poles for $s \in(-1,0)$, a consequence of Proposition 7.3 of [23].

DfFinition 4.10. - Fix $\sigma_{0}$. Let $\left\{\nu_{j}\right\}_{j=1}^{n}$ be a non-negative chain in the $\sigma_{0}$-support of $\sigma_{2}^{\vee}$. We shall say $\left\{\nu_{j}\right\}_{j=1}^{n}$ is regular if either $\nu_{1}=1 / 2$ or $\nu_{1}=0$, or $\nu_{1}=1$ and $L(s)^{-1}$ divides $L\left(s, \sigma_{0} \times \tau\right)^{-1}$ (Lemma 4.9). Observe that by [23] this last condition (on $L$-functions) implies that the representation induced from $\left(\sigma_{0} \otimes|\operatorname{det}()|\right) \otimes \tau$, i.e. $\nu \sigma_{0} \rtimes \tau$ in the notation of [34], is reducible. (See Theorem 3.3 of [34].)

DeFINITION 4.11. - Fix $\sigma_{0}$. Let $\left\{\nu_{j}\right\}_{j=1}^{n}$ and $\left\{\nu_{k}^{\prime}\right\}_{k=1}^{m}$ be a pair of non-negative chains in the $\sigma_{0}$-support of $\sigma_{2}^{\vee}$. Assume neither $\nu_{1}=1 / 2$ nor $\nu_{1}^{\prime}=1 / 2$. We then call $\left\{\nu_{j}\right\}$ and $\left\{\nu_{k}^{\prime}\right\}$ a pair of singular chains, or a singular pair, if $\nu_{1}+\nu_{1}^{\prime}=1$.

Remark. - In view of Lemma 4.6 of [34] and Theorem 8.1 of [23], either $\nu_{1}=0$ and $\nu_{1}^{\prime}=1$, or $\nu_{1}=1$ and $\nu_{1}^{\prime}=0$.

LEMMA 4.12. - If $\left\{\nu_{j}\right\}_{j=1}^{n}$ and $\left\{\nu_{k}^{\prime}\right\}_{k=1}^{m}$ are members of a singular pair, then

$$
\gamma\left(s, \nu_{1}, \ldots, \nu_{n}\right) \gamma\left(s, \nu_{1}^{\prime}, \ldots, \nu_{m}^{\prime}\right)
$$

which we shall call the $\gamma$-function for the singular pair, is non-zero for $\operatorname{Re}(s)>0$.

LEMMA 4.13. - Every chain which is neither non-negative nor non-positive is $\gamma$-equivalent to either a singular pair or a pair of regular chains starting at $1 / 2$, with $\gamma$-function given as the product of their $\gamma$-functions. 
Proof. - Let $\left\{\nu_{j}\right\}_{j=1}^{n}$ be such a chain. Then

$$
\nu_{n}>\nu_{n-1}>\ldots>\nu_{\ell} \geq 0>\nu_{\ell-1}>\ldots>\nu_{1} .
$$

The pair

$$
\left\{\nu_{\ell}, \ldots, \nu_{n}\right\} \cup\left\{-\nu_{\ell-1}, \ldots,-\nu_{1}\right\}
$$

is a pair of non-negative chains. The initial points for these are $\nu_{\ell}$ and $-\nu_{\ell-1}$, and $\nu_{\ell}-\nu_{\ell-1}=1$ implies that the pair is singular, unless $\nu_{\ell}=-\nu_{\ell-1}=1 / 2$. Moreover

$$
\gamma\left(s ; \nu_{1}, \ldots, \nu_{n}\right)=\gamma\left(s ; \nu_{\ell}, \ldots, \nu_{n}\right) \gamma\left(s ;-\nu_{\ell-1}, \ldots,-\nu_{1}\right)
$$

Then $\left\{\nu_{j}\right\}_{j=1}^{n}$ is $\gamma$-equivalent to the pair $\left\{\nu_{\ell}, \ldots, \nu_{n}\right\} \cup\left\{-\nu_{\ell-1}, \ldots,-\nu_{1}\right\}$.

DEFINITION 4.14. - Let $\left\{\nu_{j}\right\}_{j=1}^{n}$ and $\left\{\nu_{k}^{\prime}\right\}_{k=1}^{m}$ be a pair of $\sigma_{0}$-chains in the $\sigma_{0}$-support of $\sigma_{2}^{\vee}$. We shall say $\left\{\nu_{j}\right\}_{j=1}^{n}$ can be completed to $\left\{\nu_{k}^{\prime}\right\}_{k=1}^{m}$, if $m \geq n$ and $\nu_{n}=\nu_{m}^{\prime}$.

PROPOSITION 4.15. - Every non-negative chain can be completed to either a regular chain, a member of a singular pair, or a chain whose initial point is negative.

Proof. - Let $\left\{\nu_{j}\right\}_{j=1}^{n}$ be a non-negative chain. Write

$$
\gamma\left(s ; \nu_{1}, \ldots, \nu_{n}\right)=\frac{L\left(s-\nu_{n}-1\right) L\left(s+\nu_{1}-1\right)}{L\left(s-\nu_{1}\right) L\left(s+\nu_{n}\right)} .
$$

Assume $\left\{\nu_{j}\right\}$ is not of the types mentioned in the proposition and in particular if $\nu_{1}=1$, then $L(s-1)^{-1}$ does not divide $L\left(s-1, \sigma_{0} \times \tau\right)^{-1}$. By Theorem $4.4, L\left(s-\nu_{1}\right)$ must be cancelled by the $\gamma$-function of another chain $\left\{\nu_{k}^{\prime}\right\}_{k=1}^{m}$. Write

$$
\gamma\left(s ; \nu_{1}^{\prime}, \ldots, \nu_{m}^{\prime}\right)=\frac{L\left(s-\nu_{m}^{\prime}-1\right) L\left(s+\nu_{1}^{\prime}-1\right)}{L\left(s-\nu_{1}^{\prime}\right) L\left(s+\nu_{m}^{\prime}\right)} .
$$

By Lemma 4.5, we may assume that either $\nu_{1}=\nu_{m}^{\prime}+1$ or $\nu_{1}+\nu_{1}^{\prime}=1$. Suppose $\nu_{1}=\nu_{m}^{\prime}+1$. We can then complete $\left\{\nu_{j}\right\}_{j=1}^{n}$ to the chain $\left\{\nu_{j}\right\}_{j=1}^{n} \cup\left\{\nu_{k}^{\prime}\right\}_{k=1}^{m}$. Observe that

$$
\gamma\left(s ; \nu_{1}, \ldots, \nu_{n}\right) \gamma\left(s ; \nu_{1}^{\prime}, \ldots, \nu_{m}^{\prime}\right)
$$

is equal to the $\gamma$-function of $\left\{\nu_{j}\right\}_{j=1}^{n} \cup\left\{\nu_{k}^{\prime}\right\}_{k=1}^{m}$. We can therefore replace the two chains with their union and continue with the argument if $\nu_{1}^{\prime}>0$. If $\nu_{1}+\nu_{1}^{\prime}=1$ and $\nu_{1}^{\prime} \geq 0$, we then have a singular pair since we may assume $\nu_{1} \neq \nu_{1}^{\prime}$. Otherwise, we can consider $\left\{-\nu_{k}^{\prime}\right\}_{k=m}^{1}$ and then complete $\left\{\nu_{j}\right\}_{j=1}^{n}$ lo the chain $\left\{\nu_{j}\right\}_{j=1}^{n} \cup\left\{-\nu_{k}^{\prime}\right\}_{k=m}^{1}$, since $\nu_{1}=1+\left(-\nu_{1}^{\prime}\right)$. By Lemma 4.8, $\gamma\left(s ; \nu_{1}^{\prime}, \ldots, \nu_{m}^{\prime}\right)=\gamma\left(s ;-\nu_{m}^{\prime}, \ldots,-\nu_{1}^{\prime}\right)$ and therefore again $\gamma\left(s ; \nu_{1}, \ldots, \nu_{n}\right) \gamma\left(s ; \nu_{1}^{\prime}, \ldots, \nu_{m}^{\prime}\right)$ is equal to the $\gamma$-function of the union. We now use the induction if $-\nu_{m}^{\prime}>0$.

PRoposition 4.16. - Fix $\sigma_{0}$. Then every $\sigma_{0}$-chain is $\gamma$-equivalent to a chain which can be completed to a chain which is $\gamma$-equivalent to either a regular chain, a pair of regular chains, or a singular pair of chains.

Proof. - By Lemma 4.13, every chain with both positive and negative terms is $\gamma$ equivalent to either a singular pair or a pair of regular chains starting at $1 / 2$. Moreover, 
by Lemma 4.8 , every non-positive chain can be replaced by a non-negative one. Wc now apply Proposition 4.15, and Lemma 4.13, if there are any mixed chains.

We are now ready to prove Theorem 4.1.

LEMMA 4.17. - The product

$$
\prod_{i=1}^{b} L\left(s+(b+1) / 2-i-1, \sigma_{0} \times \tau\right) / L\left(s+(b+1) / 2-i, \sigma_{0} \times \tau\right)
$$

is non-zero for $\operatorname{Re}(s)>0$.

Proof. - Clear.

We now consider contributions from $\sigma_{0}$-chains.

LEMMA 4.18. - Let $\left\{\nu_{j}\right\}_{j=1}^{n}$ be a regular chain with $\nu_{1}=0$ or $1 / 2$. Then

$$
\prod_{i=1}^{b} \gamma\left(s+(b+1) / 2-i ; \nu_{1}, \ldots, \nu_{n}\right)
$$

is non-zero for $\operatorname{Re}(s)>0$.

Proof. - The product

$$
\prod_{i=1}^{b} \prod_{j=1}^{n} \frac{L\left(s+(b+1) / 2-i-\nu_{j}-1\right) L\left(s+(b+1) / 2-i+\nu_{j}-1\right)}{L\left(s+(b+1) / 2-i-\nu_{j}\right) L\left(s+(b+1) / 2-i+\nu_{j}\right)}
$$

can be written with a numerator (a polynomial in $q^{-s}$ ) as

$$
\begin{aligned}
& \prod_{i=1}^{n} L\left(s+(b+1) / 2-1-\nu_{j}\right)^{-1} L\left(s+(b+1) / 2-1+\nu_{j}\right)^{-1} \\
& =\prod_{j=1}^{n} L\left(s+(b+1) / 2-j-\nu_{1}\right)^{-1} L\left(s+(b+1) / 2-2+j+\nu_{1}\right)^{-1}
\end{aligned}
$$

We shall show that every factor which has a zero at $s$ with $\operatorname{Re}(s)>0$, cancels with a factor from the denominator.

Since $j \geq 1, b \geq 1$, and $\nu_{1} \geq 0,(b+1) / 2-2+j+\nu_{1} \geq 0$ which allows us to disregard factors

$$
\prod_{j=1}^{n} L\left(s+(b+1) / 2-2+j+\nu_{1}\right)^{-1} .
$$

We must therefore consider those factors for which

$$
(b+1) / 2-j-\nu_{1}<0
$$

or

$$
n \geq j>(b+1) / 2-\nu_{1} .
$$


Let $b_{0}$ be the first integer strictly greater than $(b+1) / 2-\nu_{1}$. We may assume $b_{0} \leq n$. The product is then over $b_{0} \leq j \leq n$, i.e. we need to consider:

$$
L\left(s+(b+1) / 2-b_{0}-\nu_{1}\right)^{-1} \ldots L\left(s+(b+1) / 2-n-\nu_{1}\right)^{-1} .
$$

The denominator is the product

$$
\prod_{j=1}^{n} L\left(s+(b+1) / 2-b-j-\nu_{1}\right)^{-1} L\left(s+(b+1) / 2-b+j-2+\nu_{1}\right)^{-1} .
$$

For $\nu_{1}=0$,

$$
b_{0}= \begin{cases}\frac{b+3}{2} & b=\text { odd } \\ \frac{b+2}{2} & b=\text { even }\end{cases}
$$

while for $\nu_{1}=1 / 2$

$$
b_{0}= \begin{cases}\frac{b+1}{2} & b=\text { odd } \\ \frac{b+2}{2} & b=\text { even }\end{cases}
$$

It is easiest to consider four cases:

Case 1. $-\nu_{1}=0$ and $b$ is odd. Then $b_{0}=\frac{b+3}{2}$ and the numerator of concern is

$$
L(s-1)^{-1} \ldots L(s-(n-(b+1) / 2))^{-1} .
$$

The denominator gives

$$
\prod_{j=1}^{n} L(s-b / 2+1 / 2-j)^{-1} L(s-b / 2-3 / 2+j)^{-1}
$$

Given an integer $\ell, 1 \leq \ell \leq n-\frac{b+1}{2}$, either $\ell \leq \frac{b+1}{2}$, in which case $j=\frac{b+3}{2}-\ell$ will satisfy $1 \leq j<n$, using $b_{0} \leq n$, and $L(s-\ell)^{-1}$ will be cancelled by $L(s-b / 2-3 / 2+j)^{-1}$; or $\frac{b+1}{2} \leq \ell \leq n-\frac{b+1}{2}$, in which case the integer $j=\ell-\frac{b-1}{2}$ satisfies $1 \leq j \leq n-b<n$ and $L(s-\ell)^{-1}$ cancels off $L(s-b / 2+1 / 2-j)^{-1}$, proving the lemma in the first case.

Case 2. $-\nu_{1}=0$ and $b$ is even. Then $b_{0}=\frac{b+2}{2}$ and the numerator is

$$
L(s-1 / 2)^{-1} \ldots L(s+(b+1) / 2-n)^{-1} .
$$

The denominator is

$$
\prod_{j=1}^{n} L(s-b / 2+1 / 2-j)^{-1} L(s-b / 2-3 / 2+j)^{-1} .
$$

Given $1 / 2 \leq \ell \leq n-\frac{b+1}{2}$, half of an odd integer, either $\ell \leq(b+1) / 2$, in which case the integer $j=(b+3) / 2-\ell$ will satisfy $1 \leq j \leq(b+1) / 2<n$, using $b_{0} \leq n$, and $L(s-\ell)^{-1}$ will be cancelled by $L(s-b / 2-3 / 2+j)^{-1}$; or $\frac{b+1}{2} \leq \ell \leq n-\frac{b+1}{2}$, in which case the integer $j=\ell-(b-1) / 2$ satisfies $1 \leq j \leq n-b<n$ and $L(s-\ell)^{-1}$ cancels $L(s-b / 2+1 / 2-j)^{-1}$. 
Case 3. $-\nu_{1}=1 / 2$ and $b$ is odd. Then $b_{0}=\frac{b+1}{2}$ and the numerator is

$$
L(s-1 / 2)^{-1} \ldots L(s+b / 2-n)^{-1},
$$

while the denominator equals

$$
\prod_{j=1}^{n} L(s-b / 2-j)^{-1} L(s-b / 2-1+j)^{-1}
$$

As in Case 2, given $1 / 2 \leq \ell \leq n-(b / 2)$, half of an odd integer, either $\ell \leq b / 2$ for which the integer $j=\frac{b+2}{2}-\ell$ satisfies $1 \leq j \leq(b+1) / 2 \leq n$ and $L(s-\ell)^{-1}$ cancels $L(s-b / 2-1+j)^{-1}$, or $b / 2<\ell \leq n-(b / 2)$, in which case the integer $j=\ell-(b / 2)$ satisfies $1 \leq j \leq n-b<n$ and $L(s-\ell)^{-1}$ cancels $L(s-(b / 2)-j)^{-1}$.

Case 4. $-\nu_{1}=1 / 2$ and $b$ is even. Then $b_{0}=(b+2) / 2$ and the numerator is

$$
L(s-1)^{-1} \ldots L(s+b / 2-n)^{-1} .
$$

Given $1 \leq \ell \leq n-(b / 2)$, an integer, either $\ell \leq b / 2$ for which the integer $j=(b+2) / 2-\ell$ will satisfy $1 \leq j \leq b / 2<n$ and $L(s-\ell)^{-1}$ will be cancelled by $L(s-b / 2-1+j)^{-1}$, or $b / 2<\ell \leq n-(b / 2)$, in which case the integer $j=\ell-(b / 2)$ satisfies $1 \leq j \leq n-b<n$ and $L(s-\ell)^{-1}$ is cancelled by $L(s-(b / 2)-j)^{-1}$.

The lemma is now complete.

LEMMA 4.19. - Let $\left\{\nu_{j}\right\}_{j=1}^{n}$ and $\left\{\nu_{k}^{\prime}\right\}_{k=1}^{m}$ be a singular pair. Then

$$
\prod_{i=1}^{b} \gamma\left(s+(b+1) / 2-i ; \nu_{1}, \ldots, \nu_{n}\right) \gamma\left(s+(b+1) / 2-i ; \nu_{1}^{\prime}, \ldots, \nu_{m}^{\prime}\right)
$$

is non-zero for $\operatorname{Re}(s)>0$.

Proof. - It is enough to prove the same statement for

$$
\prod_{i=1}^{b} L\left(s+(b+1) / 2-i-\nu_{n}-1\right) / L\left(s+(b+1) / 2-i+\nu_{n}\right),
$$

as well as for

$$
\prod_{i=1}^{b} L\left(s+(b+1) / 2-i-\nu_{m}^{\prime}-1\right) / L\left(s+(b+1) / 2-i+\nu_{m}^{\prime}\right) .
$$

Suppose $\nu_{n}>(b-2) / 2$. Then

$$
(b+1) / 2-i+\nu_{n}>b-(1 / 2)-i \geq-1 / 2 .
$$

By Theorem 8.1 of [23] and Lemma 4.6 of [34], $\nu_{n}$ is a half integer, since $\tau$ is generic. Consequently

$$
(b+1) / 2-i+\nu_{n} \geq 0
$$

proving the lemma in this case. 
Now suppose $0 \leq \nu_{n} \leq(b-2) / 2$. Given an integer $j, b \geq j>(b+1) / 2+\nu_{n}$, choose an integer $i, 2 \nu_{n}$ being an integer, such that $i=j-2 \nu_{n}-1$. Then

$$
\begin{aligned}
b \geq i=j-2 \nu_{n}-1 & >(b+1) / 2-\nu_{n}-1 \\
& \geq(b+1) / 2-(b-2) / 2-1 \\
& =1 / 2 .
\end{aligned}
$$

This implies that $i \geq 1$ since it is an integer. Consequently the factor $L(s+(b+1) / 2-$ $\left.j+\nu_{n}\right)^{-1}$ is cancelled off by $L\left(s+(b+1) / 2-i-\nu_{n}-1\right)^{-1}$. The lemma is now proved.

LEMMA 4.20. - Let $\bigcup_{\ell=1}^{c}\left\{\nu_{j}^{\ell}\right\}_{j=1}^{n_{\ell}}$ be the union of all the regular $\sigma_{0}$-chains with $\nu_{1}^{\ell}=1$, $1 \leq \ell \leq c$, which appear in the support of $\sigma_{2}^{\vee}$. Then the product of

$$
\prod_{i=1}^{b} \prod_{\ell=1}^{c} \gamma\left(s+(b+1) / 2-i,\left\{\nu_{j}^{\ell}\right\}_{j=1}^{n_{\ell}}\right)
$$

with

$$
\prod_{i=1}^{b} L\left(s+(b+1) / 2-i-1, \sigma_{0} \times \tau\right) / L\left(s+(b+1) / 2-i, \sigma_{0} \times \tau\right)
$$

is non-zero for $\operatorname{Re}(s)>0$.

Proof. - Going back over the proof of Lemma 4.9, using Theorem 4.4, it follows that in fact $L(s)^{-c}$ divides $L\left(s, \sigma_{0} \times \tau\right)^{-1}$. Set

$$
\theta\left(s, \sigma_{0} \times \tau\right)=L\left(s, \sigma_{0} \times \tau\right) / L(s)^{c} .
$$

Then both

$$
\prod_{i=1}^{b} \theta\left(s+(b+1) / 2-i-1, \sigma_{0} \times \tau\right) / \theta\left(s+(b+1) / 2-i, \sigma_{0} \times \tau\right)
$$

(Lemma 4.17) and

$$
\prod_{\ell=1}^{c} \prod_{i=1}^{b} L\left(s+(b+1) / 2-i-\nu_{n_{\ell}}^{\ell}-1\right) / L\left(s+(b+1) / 2-i+\nu_{n^{\ell}}^{\ell}\right)
$$

(Proof of Lemma 4.19) are non-zero for $\operatorname{Re}(s)>0$ and their product is equal to the product in the statement of the lemma.

Theorem 4.1 is now a consequence of Proposition 4.16, and Lemmas 4.17, 4.18, 4.19, and 4.20 , applied to every regular chain or singular pair.

REMARK 4.21. - Lemmas 4.1 and 4.6 of [34] have a similar proof which is a clever application of Casselman's square integrability criterion [5]. It extends to other classical groups which are not discussed in [34] as well, when the lemmas are formulated appropriately. The only change is in the case of unitary groups which implies $\tilde{\bar{\rho}}_{0, j} \cong \rho_{0, j}$. This is precisely what is needed for obtaining our results.

$4^{e}$ SÉRIE - TOME $31-1998-\mathrm{N}^{\circ} 4$ 
REMARK 4.22. - The regular chains and singular pairs defincd here are the samc as those defined by Tadić in [34] which is the same as in [5], and that is how we chose these terminologies. In fact our Proposition 4.16 proves some of the results of [34]. Observe that Proposition 4.16 is based on Theorem 4.4 which was proved in [23].

Remark 4.23. - Muić [42] now also has a proof of Theorem 4.1 when $\mathbf{G}=S p_{2 n}$ or $S O_{2 n+1}$. His proof, although quite different, also relies on the results of [23]. The paper contains some very interesting results for these groups.

(See the introduction here.)

\section{Applications}

In this section we prove a result which determines the poles of intertwining operators in terms of those of $L$-functions whenever injectivity (Definition 3.1) holds in a certain level. We then apply this result to determine the poles of intertwining operators in terms of Artin $L$-functions in an important archimedean case (Theorem 6.1, Section 6).

Let $\mathbf{G}$ be again a quasisplit connected reductive algebraic group over a local field $F$ of characteristic zero as in Sections 1-3. Fix a Borel subgroup $\mathbf{B}$ and write $\mathbf{B}=\mathbf{T U}$, where $\mathbf{T}$ is a maximal torus and $\mathbf{U}$ denotes the unipotent radical of $\mathbf{B}$.

Fix a $F$-parabolic subgroup $\mathbf{P}=\mathbf{M N}$ with $\mathbf{N} \subset \mathbf{U}$ and $\mathbf{T} \subset \mathbf{M}$, a Levi decomposition. Let $\mathbf{A}_{0}, W\left(\mathbf{A}_{0}\right), \psi, \psi_{M}, \mathfrak{a}^{*}, \mathfrak{a}_{\mathfrak{C}}^{*}$, all be as in Section 1 . Suppose $\pi$ is an irreducible

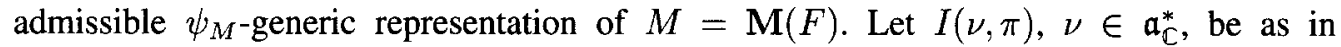
Section 1.

Assume $\mathbf{P}$ is maximal and let $\alpha$ be the unique simple root in $\mathbf{N}$. As in [23], let $\widetilde{\alpha}=\langle\rho, \alpha\rangle^{-1} \cdot \rho$, where $\rho$ is half the sum of roots in $\mathbf{N}$. Given $s \in \mathbb{C}, s \tilde{\alpha} \in \mathfrak{a}_{\mathbb{C}}^{*}$. Let $A\left(s \tilde{\alpha}, \pi, w_{0}\right)$ be the standard intertwining operator from $I(s \widetilde{\alpha}, \pi)$ into $I\left(w_{0}(s \widetilde{\alpha}), w_{0}(\pi)\right)$, where $w_{0}$ is a representative for $\widetilde{w}_{0}$.

As in Section 1 , denote by ${ }^{L} M$, the $L$-group of $\mathrm{M}$ and let ${ }^{L} \mathfrak{n}$ be the Lie algebra of the $L$-group of $\mathbf{N}$. Let $r$ be the adjoint action of ${ }^{L} M$ on ${ }^{L} \mathfrak{n}$ and decompose $r=\bigoplus_{i=1}^{m} r_{i}$, with ordering as in [23]. For each $i, 1 \leq i \leq m$, let $L\left(s, \sigma, r_{i}\right)$ be the local $L$-function defined in [23]. (See Section 1 here.) It is defined to agree completely with Langlands definition of $L$-functions whenever there is a parametrization. In particular the $L$-function for arbitrary $\sigma$ is just the analytic continuation of the one attached to the tempered inducing data through the product formula (cf. part 3 of Theorem 3.5 and equation 7.10 of [23]). (See also Theorem 5.2 of [26].)

Next, embed $\pi$ as a subrepresentation of a module

$$
I_{M}(\nu, \sigma)=\operatorname{Ind}_{M_{\theta} N_{\theta} \uparrow M} \sigma_{\nu} \otimes 1,
$$

where $\sigma$ is in the discrete series and $\nu$ is in the closure of the negative Weyl chamber of $\mathfrak{a}_{\theta}$ (Langlands classification). Here $\mathfrak{a}_{\theta}$ is the real Lie algebra of the split component $\mathbf{A}_{\theta}$ of the center of $\mathbf{M}_{\theta}$.

As usual fix $s \in \mathbb{C}$. Embed $I(s \tilde{\alpha}, \pi) \hookrightarrow I(s \widetilde{\alpha}+\nu, \sigma)$, where $s \widetilde{\alpha}$ in $I(s \tilde{\alpha}+\nu, \sigma)$ denotes an extension of $s \widetilde{\alpha}$ to $\mathfrak{a}_{\theta, C}^{*}$. Let $\widetilde{w}_{0}$ denote the longest element in the Weyl group of $\mathbf{A}_{0}$ in $\mathbf{G}$ modulo that of $\mathbf{A}_{0}$ in $\mathbf{M}$. Fix a reduced decomposition $\widetilde{w}_{0}=\widetilde{w}_{n-1} \ldots \widetilde{w}_{1}$ with respect 
to the Levi subgroup $\mathbf{M}_{\theta}$ of $\mathbf{G}$ (Lemma 2.1.1 of [25]). For each $j, 2 \leq j \leq n-1$, there exists a unique simple root $\alpha_{j}$ such that $\widetilde{w}_{j}\left(\alpha_{j}\right)<0$. Let $\bar{w}_{j}=\widetilde{w}_{j-1} \ldots \widetilde{w}_{1}$ with $\bar{w}_{1}=1$. Let $\Omega_{j}=\theta_{j} \cup\left\{\alpha_{j}\right\}$, where $\theta_{1}=\theta$ and $\theta_{j+1}=\widetilde{w}_{j}\left(\theta_{j}\right), 1 \leq j \leq n-1$. The group $\mathbf{M}_{\Omega_{j}}$ contains $\mathbf{M}_{\theta_{j}}$ as the Levi subgroup of a maximal parabolic subgroup. For each $j, \sigma_{j}=\bar{w}_{j}\left(\sigma_{\nu}\right)$ is an unramified twist of a discrete series of $M_{\theta_{j}}$. Let $I_{\Omega_{j}}\left(\sigma_{j}\right)$ denote the corresponding induced representation of $M_{\theta_{j}}$. Write $I_{\Omega_{j}}\left(\sigma_{j}\right)=I\left(\nu_{j}, \sigma_{j}^{\prime}\right)$, where $\sigma_{j}^{\prime}$ is in the discrete series. Up to an unramified twist $\sigma_{j}^{\prime}$ is unique. We will assume that for each $j, \sigma_{j}^{\prime}$ is such that every standard module of $M_{\Omega_{j}}$ which has $\sigma_{j}^{\prime}$ as its tempered inducing data satisfies injectivity (Definition 3.1).

THEOREM 5.1. - Suppose Conjecture 1.1 is valid whenever F is p-adic, e.g. G is classical. Moreover assume for each $j$ every standard module of $M_{\Omega_{j}}$ which has $\sigma_{j}^{\prime}$ as its tempered inducing data satisfies injectivity (Definition 3.1). Then

$$
\prod_{i=1}^{m} L\left(i s, \tilde{\pi}, r_{i}\right)^{-1} A\left(s \tilde{\alpha}, \pi, w_{0}\right)
$$

is entire.

Proof. - The intertwining operator $A\left(s \widetilde{\alpha}, \pi, w_{0}\right)$ is a restriction of the product of rank one operators $A\left(\nu_{j}, \sigma_{j}^{\prime}, \tilde{w}_{j}\right), 1 \leq j \leq n-1$. So are the $L$-functions $L\left(s, \tilde{\pi}, r_{i}\right)$, of course under validity of Conjecture 1.1 if $F$ is non-archimedean (Part 3 of Theorem 3.5 and equation (7.10) of [23], as well as Theorem 5.2 of [26]). One must therefore prove the following lemma.

THEOREM 5.2. - Theorem 5.1 is valid if $\pi$ is in the discrete series.

Proof. - We need to show that if $I(s \widetilde{\alpha}, \pi)$ satisfies injectivity for all $s \in \mathbb{C}$, with $\operatorname{Re}(s)>0$, then the theorem is valid.

If $\operatorname{Re}(s)>0$, then $A\left(s \tilde{\alpha}, \pi, w_{0}\right)$ and $\prod_{i=1}^{m} L\left(i s, \tilde{\pi}, r_{i}\right)$ are both holomorphic. For $s$ with $\operatorname{Re}(s)=0$ which is a pole of $A\left(s \widetilde{\alpha}, \pi, w_{0}\right), I(s \widetilde{\alpha}, \pi)$ is irreducible by the theory of $R$-groups. Consequently the local coefficient $C_{\psi}(s \widetilde{\alpha}, \pi)$ must have a zero of the same multiplicity (cf. equation (1.2) of [23] and Section 1 here). Since $\prod_{i=1}^{m} L\left(1-i s, \pi, r_{i}\right)$ is holomorphic for $\operatorname{Re}(s)=0$, the same is true about $\prod_{i=1}^{m} L\left(i . s, \tilde{\pi}, r_{i}\right)^{-1}$. It remains to consider the case of $\operatorname{Re}(s)<0$.

Suppose $\operatorname{Re}(s)>0$. Given $f$ in an irreducible subspace of $I(s \tilde{\alpha}, \pi)$, there exists a $\psi$ extending $\psi_{M}$ such that $\lambda_{\psi}(s \tilde{\alpha}, \pi)(f) \neq 0$ by injectivity assumption. Thus it follows from

$$
\lambda_{\psi}(s \widetilde{\alpha}, \pi)=C_{\psi}(s \widetilde{\alpha}, \pi) \lambda_{\psi}\left(w_{0}(s \widetilde{\alpha}), w_{0}(\pi)\right) A\left(s \widetilde{\alpha}, \pi, w_{0}\right)
$$

and equation (3.11) of Theorem 3.5 of [23] that $C_{\psi}(s \widetilde{\alpha}, \pi) A\left(s \widetilde{\alpha}, \pi, w_{0}\right)$ must never be zero for $\operatorname{Re}(s)>0$. Using

$$
C_{\psi}\left(w_{0}(s \widetilde{\alpha}), w_{0}(\pi)\right) A\left(w_{0}(s \widetilde{\alpha}), w_{0}(\pi), w_{0}^{-1}\right) C_{\psi}(s \widetilde{\alpha}, \pi) A\left(s \widetilde{\alpha}, \pi, w_{0}\right)=I
$$

it is now clear that

$$
\left.C_{\psi}\left(w_{0}(s \widetilde{\alpha}), w_{0}(\pi)\right) A\left(w_{0}(s \widetilde{\alpha}), w_{0}(\pi), w_{0}^{-1}\right)\right)
$$

$4^{\text {e }}$ SÉRIE - TOME $31-1998-\mathrm{N}^{\circ} 4$ 
is holomorphic for $\operatorname{Re}(s)>0$. This implies that

$$
\prod_{i=1}^{m} L\left(1-i s, \pi, r_{i}\right) / L\left(i s, \tilde{\pi}, r_{i}\right) \cdot A\left(s \widetilde{\alpha}, \pi, w_{0}\right)
$$

is holomorphic whenever $\operatorname{Re}(s)<0$, completing the lemma.

With respect to reducibility of standard modules one has the following

Proposition 5.3. - Let $F$ be any local field. But in the case of p-adic $F$, assume that $\mathbf{G}$ satisfies Conjecture 1.1. Let $\mathbf{P}=\mathbf{M N}$ be a maximal parabolic subgroup of $\mathbf{G}$ and fix a generic irreducible tempered representation $\sigma$ of $M$. Suppose $\operatorname{Re}(s)>0$. Assume Vogan's theorem is valid for every $I(s \widetilde{\alpha}, \sigma)$, i.e. if $J(s \widetilde{\alpha}, \sigma)$ is generic, then $I(s \widetilde{\alpha}, \sigma)$ is irreducible. Then $I(s \widetilde{\alpha}, \sigma)$ is irreducible if and only if $\prod_{i=1}^{m} L\left(1-i s, \sigma, r_{i}\right)^{-1} \neq 0$. When $F$ is archimedean, the L-functions are those of Artin attached by Langlands and the assumption is already a theorem (Theorem 6.1 of [37]).

Proof. - If $I(s \widetilde{\alpha}, \sigma)$ is reducible, then by our assumption (Vogan's Theorem 6.1 of [37] if $F$ is archimedean) its Langlands quotient is not generic. Consequently

$$
\lambda_{\psi}\left(w_{0}(s \widetilde{\alpha}), w_{0}(\sigma)\right) A\left(s \widetilde{\alpha}, \sigma, w_{0}\right)
$$

will be identically zero. But $\lambda_{\psi}(s \widetilde{\alpha}, \sigma)$ is not zero. Consequently

$C_{\psi}\left(s \widetilde{\alpha}, \sigma, w_{0}\right)^{-1}=0$ which implies $\prod_{i=1}^{m} L\left(1-i s, \sigma, r_{i}\right)^{-1}=0$ (equations (3.11) and (7.4) of [23]) since $\prod_{i=1}^{m} L\left(i s, \sigma, \tilde{r}_{i}\right)^{-1}$ is non-zero for $\operatorname{Re}(s)>0$.

Conversely suppose $I(s \widetilde{\alpha}, \sigma)$ is irreducible. Then $\lambda_{\psi}\left(w_{0}(s \widetilde{\alpha}), w_{0}(\sigma)\right) A\left(s \widetilde{\alpha}, \sigma, w_{0}\right)$ is never zero and therefore $\prod_{i=1}^{m} L\left(1-i s, \sigma, r_{i}\right)^{-1} \neq 0$ since $\lambda_{\psi}(s \widetilde{\alpha}, \sigma)$ is holomorphic.

More generally we have the following conditional reducibility criteria for representations induced from irreducible generic quasi-tempered representations. Applying standard arguments, such as inducing in stages for singular parameters, we may assume that their complex parameters are in the positive Weyl chamber.

Proposition 5.4. - Let $F$ be any local field. Suppose Vogan's theorem is valid for the standard modules of $G$. Let $\mathbf{P}=\mathbf{M N}$ be an arbitrary parabolic subgroup of $\mathbf{G}, \mathbf{P} \supset \mathbf{B}$, and fix an irreducible $\chi$-generic tempered representation of $M$. Let $\nu \in \mathfrak{a}_{\complement}^{*}$ be in the positive Weyl chamber of the split component of $\mathbf{M}$. Let $C_{\psi}(\nu, \sigma)$ be the local coefficient attached to $\nu, \sigma$, and $\psi$. Then $I(\nu, \sigma)$ is irreducible if and only if $C_{\psi}(\nu, \sigma)^{-1} \neq 0$.

Proof. - Exactly as in Proposition 5.3.

\section{An Important Archimedean Case}

In this section we will apply Theorem 5.1 to an important special case when $F$ is archimedean. The case in hand has an important application in lifting of automorphic forms from classical groups to $G L_{r}$ as being pursued in [11,30,31], using the converse theorem [9]. 
More precisely, let $F=\mathbb{R}$ or $\mathbb{C}$ and let $\mathbf{G}=S O_{2 n}$, the split special orthogonal group of rank $n$. We will be concerned only with its Siegel parabolic subgroup $\mathbf{P}=\mathbf{M N}$ for which $\mathbf{M} \cong G L_{n}$. Let $\sigma$ be an irreducible admissible generic representation of $M=G L_{n}(F)$.

Let $W_{F}$ be the Weil group of $\bar{F} / F$ (cf. $[1,17,18,24]$ ) and fix a representation

$$
\phi: W_{F} \rightarrow{ }^{L} M=G L_{n}(\mathbb{C})
$$

which parametrizes $\sigma$ as in [17]. Given a representation $r$ of ${ }^{L} M=G L_{n}(\mathbb{C})$, let $L(s, r \cdot \phi)$ be the Artin $L$-function attached to the representation $r \cdot \phi$ of $W_{F}$. (See $[1,18,24]$.) Finally, note that the adjoint action of ${ }^{L} M=G L_{n}(\mathbb{C})$ on ${ }^{L} \mathfrak{n}$, the Lie algebra of the $L$-group of $\mathbf{N}$, is equal to $\Lambda^{2} \rho_{n}$, the exterior square representation of $G L_{n}(\mathbb{C})$ (cf. [27]). We shall prove:

Theorem 6.1. - Let $\mathbf{G}=S O_{2 n}$ and $F=\mathbb{R}$ or $\mathbb{C}$. Assume $\mathbf{P}=\mathbf{M N}$ is the Siegel parabolic subgroup of $\mathrm{G}$. Let $\sigma$ be an irreducible admissible generic representation of $M$. Choose the homomorphism (representation)

$$
\phi: W_{F} \rightarrow G L_{n}(\mathbb{C})
$$

parametrizing $\sigma$. Let $A\left(s \tilde{\alpha}, \sigma, w_{0}\right)$ be the standard intertwining operator discussed before. Then as a function of $s$

$$
L\left(s, \Lambda^{2} \rho_{n} \cdot \tilde{\phi}\right)^{-1} A\left(s \tilde{\alpha}, \sigma, w_{0}\right)
$$

is entire, where $\tilde{\phi}$ is the contragredient of $\phi$.

Proof. - By Vogan's results (Theorem 6.2.f of [37]) and the fact that $R$-groups for $G L_{n}$ are trivial, one concludes that $\sigma$ is in fact a full induced representation, induced from a tensor product of essentially discrete series representations of a product $\prod_{i} G L_{m_{i}}(F)$. Since $F=\mathbb{R}$ or $\mathbb{C}, m_{i}=1$ or 2 .

Going back to Theorem 5.1, we only need to prove that the injectivity holds in each of the rank one cases. Then $G$ is either $G L_{m}(\mathbb{R}), m=2,3,4, G L_{2}(\mathbb{C})$, or finally split $S O_{4}(\mathbf{R})$. The Levi subgroups for $m=3$ and 4 are $G L_{2} \times G L_{1}$ and $G L_{2} \times G L_{2}$, respectively, while that of $\mathrm{SO}_{4}$ is the Levi subgroup of the Siegel parabolic subgroup, i.e. $\mathbf{M} \cong G L_{2}$. The $L$-functions in [23] are now precisely those of Artin mentioned above (Theorem 3.5 of [23]).

We shall now check the injectivity in each of the above cases. More precisely, we must show that in each case, every representation induced from an essentially discrete series data whose central character is in the positive Weyl chamber, i.e. a standard representation, contains no non-generic irreducible subspaces.

When $\mathbf{G}=G L_{2}$ this is well known. Suppose $G=S O_{4}(\mathbf{R})$. Realize the (topological) connected component of $G$ as the quotient of $S L_{2}(\mathbb{R}) \times S L_{2}(\mathbb{R})$ by $\{ \pm 1\}$. The (topological) connected component of $M=G L_{2}(\mathbb{R})$ is the image of $S L_{2}(\mathbb{R}) \times \mathbb{R}^{*}$ with $\mathbb{R}^{*}$ realized as the diagonal subgroup of the second $S L_{2}(\mathbb{R})$ in this product. The induction corresponds to a principal series in $S L_{2}(\mathbb{R})$. The injectivity is then a consequence of the same fact for $S L_{2}(\mathbf{R})$.

It remains to consider $G L_{m}(\mathbb{R})$ with $m=3$ or 4 . The result must be contained somewhere in Speh's thesis [32]. In fact $m=3$ is clearly there. But, one expects it to be valid for any standard module of $G L_{m}(\mathbf{R})$ for arbitrary $m$, and this is in fact the case and a proof of it 
was first communicated to us by Vogan. Here we include his unpublished proof of this fact in which any inaccuracy or shortfall is our responsibility and none his. We are indebted to him for providing us with a proof. Later, we realized, that there is also an analytic proof, based on the theory of canonical models [6, 40], due to Jacquet and Shalika [13].

Theorem 6.2 (Vogan, Jacquet-Shalika [13]). - Let I be a standard module of $G=G L_{n}(\mathbf{R})$. Then every non-zero irreducible subrepresentation of $I$ is large, i.e. generic. In particular, I has a unique irreducible subrepresentation.

Proof (Vogan). - Let $\mathfrak{g}$ be the complexified Lie algebra of $G$. Let $K$ be a maximal compact subgroup of $G$. We need Jantzen-Zuckerman translation functors $\psi$ and $\phi$ on the category of $(\mathfrak{g}, K)$-modules of finite length. Then

$$
\operatorname{Hom}_{\mathfrak{g}, K}(U, \psi V) \cong \operatorname{Hom}_{\mathfrak{g}, K}(\phi U, V)
$$

for any pair $(U, V)$ of finite length $(\mathfrak{g}, K)$-modules, i.e. $\phi$ is the adjoint of $\psi$. Similarly we have

$$
\operatorname{Hom}_{\mathfrak{g}, K}(U, \phi V) \cong \operatorname{Hom}_{\mathfrak{g}, K}(\psi U, V) .
$$

(See $\S 4.5$ and $\S 7.4$ of [39].) Throughout the proof, we shall call (6.2.1) and (6.2.2), the adjointness property. The proof is in several steps.

We first reduce to the regular infinitesimal characters. Let $X$ be a standard module with a singular infinitesimal character. Let $Z$ be a standard module with a regular infinitesimal character such that $X=\psi(Z)$. Fix an irreducible subrepresentation $W$ of $X$. Then by adjointness there is a non-zero $(\mathfrak{g}, K)$-map from $\phi(W)$ into $Z$. Assuming the theorem for $Z$, this implies that $\phi(W)$ has a large composition factor. But then $W$ itself must be large since tensoring with a finite-dimensional representation does not change largeness.

From now on we shall assume $X$ to have a regular infinitesimal character which we shall fix throughout. Suppose that $X=X(\gamma)$ for a regular character $\gamma$ of $H$, a $\theta$-stable Cartan subgroup of $G$ with a $\theta$-stable Lie algebra $\mathfrak{h}$. Here $\theta$ is a fixed Cartan involution fixing the Lie algebra of $K$. (See [39] for notation and terminology.) The proof will proceed by induction on the size of the $A$-parameter of the inducing data (with respect to the norm coming from the Killing form) of the standard module $X$ (for our fixed regular infinitesimal character). Assume the size of $A$-parameter is not minimal. Then by Proposition 8.2.7 of [39] there is a standard representation $Y$ with the same infinitesimal character as $X$ and a translation to a complex root-wall functor $\psi$ with adjoint $\phi$ such that

$$
0 \rightarrow X \rightarrow \phi \psi(Y) \rightarrow Y \rightarrow 0
$$

The standard representation $Y$ then has a smaller size $A$-parameter. Now suppose $W$ is an irreducible subrepresentation of $X$. Then it is one of $\phi \psi(Y)$. Applying adjointness to the non-zero map

$$
W \rightarrow \phi \psi(Y)
$$

implies the existence of a non-zero map from $\phi \psi(W)$ into $Y$. Consequently $\phi \psi(W)$ contains as a subquotient an irreducible subrepresentation of $Y$ which by induction is large. Again $W$ must be large. 
It remains to prove the theorem when the size of the $A$-parameter is minimal. Here we need to use Zuckerman derived functors and finally the fact that $G=G L_{n}(\mathbb{R})$.

Now assume that the size of the $A$-parameter of $X$ is minimal. Let $\mathfrak{q}=\mathfrak{l}+\mathfrak{u}$ be the $\theta$-stable parabolic subalgebra of $\mathfrak{g}$ defined as follows. The subalgebra $\mathfrak{l}$ is the Lie algebra of the levi subgroup $L$ which centralizes the Lie algebra $\mathfrak{t}$ of $T=H \cap K$ in $G$. The Cartan subgroup $H$ is then maximally split in $L$ and the roots of $\mathfrak{h}$ in $\mathfrak{l}$ are exactly the real roots of $\mathfrak{h}$ in $\mathfrak{g}$. To define $\mathfrak{u}$, fix a generic element $Z$ of $\mathfrak{t}$, i.e. one that vanishes only on the real roots of $\mathfrak{h}$ in $\mathfrak{g}$. Let $\mathfrak{u}$ be spanned by the root spaces of roots $\alpha$ such that

$$
\langle\alpha, \bar{\gamma} \mid \mathfrak{t}\rangle>0
$$

or

$$
\langle\alpha, \bar{\gamma} \mid \mathfrak{t}\rangle=0
$$

and $\alpha(Z)>0$, where $\bar{\gamma}$ is the infinitesimal character of $X(\gamma)$. We need

Lemma 6.3. - Suppose the A-parameter of $X(\gamma)$ is minimal. Let $\bar{\gamma}$ be the infinitesimal character of $X(\gamma)$. Then:

(6.3) Every non-real positive integral root $\alpha$ for $\bar{\gamma}$ is a root in $u$.

Proof. - Suppose

$$
\left\langle\bar{\gamma}, \alpha^{\vee}\right\rangle=m
$$

is a positive integer for some non-real root $\alpha$, where

$$
\alpha^{\vee}=2 \alpha /\langle\alpha, \alpha\rangle
$$

is the corresponding coroot and $\langle, \quad\rangle$ is the Killing form. By definition $\left\langle\gamma, \alpha^{\vee}\right\rangle=$ $\left\langle\bar{\gamma}, \alpha^{\vee}\right\rangle=m$. If $\langle\theta \alpha, \bar{\gamma}\rangle \geq 0$, then

$$
2\langle\alpha, \bar{\gamma} \mid \mathfrak{t}\rangle=\langle\alpha+\theta \alpha, \bar{\gamma}\rangle>0
$$

and thus $\alpha$ is in $u$. Assume $\langle\theta \alpha, \bar{\gamma}\rangle=\langle\theta \alpha, \gamma\rangle<0$. After changing the roles of $\alpha$ and $\theta \alpha$, if necessary, we may assume

$$
\left\langle\gamma,-\theta \alpha^{\vee}\right\rangle \geq m
$$

The parameter $\gamma^{\prime}=\gamma-m \alpha$ is a regular character with the same infinitesimal character as $\gamma$. Moreover, the (squared) length of the corresponding $A$-parameter is one fourth of

$$
\left\|\gamma^{\prime}-\theta \gamma^{\prime}\right\|^{2}=\|\gamma-\theta \gamma-m(\alpha-\theta \alpha)\|^{2}
$$

By two-dimensional geometry

$$
\left\|\gamma^{\prime}-\theta \gamma^{\prime}\right\|<\|\gamma-\theta \gamma\|
$$

if and only if

$$
2\langle\gamma-\theta \gamma, \alpha-\theta \alpha\rangle>m\|\alpha-\theta \alpha\|^{2}
$$

which we shall now prove, getting a contradiction to minimality of $\|\gamma-\theta \gamma\|$.

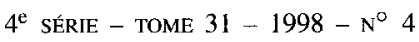


The left hand side of (6.3.2) is equal to

$$
\begin{aligned}
4\langle\gamma, \alpha\rangle-4\langle\gamma, \theta \alpha\rangle & \geq 2 m\langle\alpha, \alpha\rangle+2 m\langle\alpha, \alpha\rangle \\
& =4 m\langle\alpha, \alpha\rangle,
\end{aligned}
$$

using (6.3.1). Now the right hand side of (6.3.3) can be written as

$$
4 m\|1 / 2(\alpha+\theta \alpha)\|^{2}+4 m\|1 / 2(\alpha-\theta \alpha)\|^{2}>m\|\alpha-\theta \alpha\|^{2}
$$

since $\alpha$ is not real, proving (6.3.2) and completing the lemma.

Let $\gamma_{\mathfrak{q}}$ be, for example, as in Lemma 8.1.2 of [39], and let $X_{L}=X_{L}\left(\gamma_{\mathfrak{q}}\right)$, the standard representation of $L$, defined by $\gamma_{\mathrm{q}}$. Then by Zuckerman's cohomological induction (cf. [39]), $X=\mathcal{R}^{S} X_{L}$, where $\mathcal{R}^{S}$ is the appropriate exact cohomological functor of Zuckerman (Lemma 6.3 here, Definition 6.5.1, Corollary 8.1.15, and Situation 9.3.8 of [39], and Proposition 4.11 of [38]).

By Theorem 6.2.e of [37] all the irreducible subspaces of $X_{L}=X_{L}\left(\gamma_{\mathfrak{q}}\right)$ which is a minimal principal scrics (roots of $\mathfrak{h}$ in $\mathfrak{l}$ arc rcal roots of $\mathfrak{h}$ in $\mathfrak{g}$ and $H$ is split in $L$ ) are large.

It remains to show that for $G=G L_{n}(\mathbb{R})$, the functor $\mathcal{R}^{S}$ preserves largeness.

Let $G$ be a quasisplit real (linear) group and let $\pi$ be an irreducible admissible representation of $G$. Then $\pi$ is given as the Langlands quotient $\bar{X}(\gamma)$ of a standard module $X(\gamma)$, where $\gamma$ is a character of a $\theta$-stable Cartan subgroup $H$ whose complexified Lie algebra is $\mathfrak{h}$. Let $R(\bar{\gamma})$ be the set of integral roots of $\mathfrak{h}$ in $\mathfrak{g}$ with respect to $\bar{\gamma}$, the infinitesimal character of $X(\gamma)$ which we may assume to be regular. Let $R^{+}(\bar{\gamma})$ be the set of positive roots in $R(\bar{\gamma})$. By [37], $\pi$ is large if (cf. Definition 8.6.5 and Theorem 8.6.6 of [39] and Theorem 6.2.f of [37]):

1) If $\alpha \in R^{+}(\bar{\gamma})$ is a simple non-real root, then $\theta \alpha \in R^{+}(\bar{\gamma})$.

2) If $\alpha \in R^{+}(\bar{\gamma})$ is a simple real root, then $\alpha$ fails to satisfy the parity condition defined in Definition 8.3.11 of [39].

3) If $\alpha \in R^{+}(\bar{\gamma})$ is a simple imaginary root, then $\alpha$ is non-compact.

Suppose now that $Y$ is an irreducible subrepresentation of $X=\mathcal{R}^{S} X_{L}$. By Lemma 6.3, $\mathcal{R}^{S}$ restricted to the infinitesimal character $\bar{\gamma}_{\mathfrak{q}}$ is exact and carries irreducible representations to irrcducible representations. Therefore there is an irreducible subrepresentation $Y_{L}$ of $X_{L}$ with $Y=\mathcal{R}^{S} Y_{L}$. As we discussed above (Theorem 6.2.e of [37]), $Y_{L}$ is large. Write $Y_{L}=\bar{X}_{L}\left(\gamma_{\mathfrak{q}}^{\prime}\right)$. Since $Y_{L}$ has infinitesimal character $\bar{\gamma}_{\mathfrak{q}}^{\prime}$, the corrcsponding regular character $\gamma^{\prime}$ for $G$ satisfies (6.3) as $\gamma$ and $Y=\bar{X}\left(\gamma^{\prime}\right)$. We need to check the conditions 1)-3) for $Y$ to be large. Because of (6.3), $R^{+}\left(\bar{\gamma}^{\prime}\right)=R^{+}\left(\bar{\gamma}_{\mathfrak{q}}^{\prime}\right) \cup R_{\mathfrak{u}}^{+}\left(\bar{\gamma}^{\prime}\right)$, wherc $R_{\mathfrak{u}}^{+}\left(\bar{\gamma}^{\prime}\right)$ is the set of integral roots in $\mathfrak{u}$ with respect to $\bar{\gamma}^{\prime}$. Clearly $R_{\mathfrak{u}}^{+}\left(\bar{\gamma}^{\prime}\right)$ contains no real roots ( $u$ is $\theta$-stable) and is $\theta$-stable. But simple roots of $R^{+}\left(\bar{\gamma}^{\prime}\right)$, are then those of $R^{+}\left(\bar{\gamma}_{\mathfrak{q}}^{\prime}\right)$ plus a subset of $R_{\mathfrak{u}}^{+}\left(\bar{\gamma}^{\prime}\right)$. It is therefore clear that conditions (1) and (2) above for $Y$ is inherited from $Y_{L}$. In the case of $G L_{n}(\mathbb{R})$, every imaginary root is non-compact and therefore condition 3 ) is empty. The theorem is now complete. 


\section{REFERENCES}

[1] J. ARTHUR, Intertwining operators and residues I. Weighted characters, (J. Funct. Anal., Vol. 84, 1989, pp. 19-84).

[2] D. BARBASCH and A. MOY, Whittaker models with an Iwahori fixed vector, Representation theory and analysis on homogeneous spaces, (AMS, Rhode Island 1994, pp. 101-105).

[3] I. N. BERNSTEIN and A. V. ZELEVINSKY, Induced representations of reductive p-adic groups I, (Ann. Scient. Éc. Norm. Sup., Vol. 10, 1977, pp. 441-472).

[4] A. Borel and N. Wallach, Continuous Cohomology, Discrete Subgroups, and Representations of Reductive Groups, (Annals of Math. Studies, Vol. 94, 1980, Princeton University Press, Princeton).

[5] W. CASSELMAN, Introduction to the theory of admissible representations of p-adic reductive groups, preprint.

[6] W. Casselman, Canonical extensions of Harish-Chandra modules, (Cand. J. Math., Vol. 41, 1989, pp. 315438).

[7] W. CASSElman, Letter to Harish-Chandra, November 1982.

[8] W. Casselman and J.A. Shalika, The unramified principal series of p-adic groups II. The Whittaker function, (Comp. Math., Vol. 41, 1980, pp. 207-231).

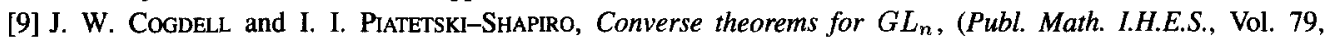
1994, pp. 157-214).

[10] S. FRIEDBERG and D. GOLDBERG, On local coefficients for nongeneric representations of some classical groups, Comp. Math., to appear.

[11] S. Gelbart, I. I. PiateTSKI-ShaPIRo, and S. Rallis, Explicit construction of automorphic L-functions, (Lecture Notes in Math 1254, Springer-Verlag, 1987).

[12] HARISH-CHANDRA, Harmonic analysis on real reductive groups III. The Maass-Selberg relations and the Plancherel formula, (Annals of Math., Vol. 104, 1976, pp. 117-201).

[13] H. JACQUET and J. A. Shalika, Rankin Selberg Convolutions: Archimedean theory, in Festschrift in Honor of I.I. Platetski-Shapiro, Part I, Editors: S. Gelbart, R. Howe, and P. SaRnaK, (Israel Math. Conf. Proc., Vol. 2, Weizmann, Jerusalem, 1990, pp. 125-207).

[14] H. JACQUET and J. A. SHALIKA, The Whittaker models for induced representations, (Pacific J. Math., Vol. 109, 1983, pp. 107-120).

[15] H. KIM, Residual spectrum for $S p_{1}$, (Comp. Math., Vol. 99, 1995, pp. 129-151).

[16] A.W. KNAPP and E.M. STEIN, Intertwining operators for semisimple groups II, (Invent. Math., Vol. 60, 1980, pp. 9-84).

[17] R. P. LANGLANDS, On the classification of irreducible representations of real algebraic groups, in (Representation Theory and Harmonic Analysis on Semisimple Lie Groups, Editors P.J. SALly, Jr. and D.A. VoGAN, Mathematical Surveys and Monographs, AMS, Vol., Vol. 31, 1989, pp. 101-170).

[18] R. P. Langlands, On Artin's L-functions, (Rice University Studies, Vol. 56, 1970, pp. 23-28).

[19] R. P. LANGLANDS, On the functional equations satisfied by Eisenstein series, (Lecture Notes in Math., Vol. 544, Springer-Verlag, 1976).

[20] J.-S. LI, Some results on the unramified principal series of p-adic groups, (Math. Ann., Vol. 292, 1992, pp. 747 761).

[21] C. Moeglin and J.-L. WaldSPurger, Le spectre résiduel de $G L(n)$, (Ann. Scient. Éc. Norm. Sup., Vol. 22, 1989, pp. 605-674).

[22] M. REEDER, p-adic Whittaker functions and vector bundles on flag manifolds, (Comp. Math., Vol. 85, 1993, pp. 9-36).

[23] F. SHAHIDI, A proof of Langlands' conjecture on Plancherel measures; Complementary series for p-adic groups, (Ann. of Math., Vol. 132, 1990, pp. 273-330).

[24] F. SHAHid, Local coefficients as Artin factors for real groups, (Duke Math. J., Vol. 52, 1985, pp. 973-1007).

[25] F. SHAHIDI, On certain L-functions, (Amer. J. Math., Vol. 103, 1981, pp. 297-356).

[26] F. SHAHIDI, On multiplicativity of local factors, in (Festschrift in Honor of I.I. Piatetski-Shapiro, Part II, Editors: S. Gelbart, R. Howe, and P. Sarnak, Israel Math. Conf. Proc., Vol. 3, Weizmann, Jerusalem, 1990, pp. 279-289).

[27] F. SHAHIDI, Twisted endoscopy and reducibility of induced representations for p-adic groups, (Duke Math. J., Vol. 66, 1992, pp. 1-41).

[28] A. SILBERGER, The Langlands quotient theorem for p-adic groups, (Math. Ann., Vol. 236, 1978, pp. 95-104).

[29] A. Silberger, Introduction to Harmonic Analysis on Reductive p-adic Groups, Math. Notes of Princeton University Press, Vol. 23, Princeton, 1979.

$4^{\text {e }}$ SÉRIE - TOME $31-1998-\mathrm{N}^{\circ} 4$ 
[30] D. SOUDRY, Rankin-Selberg convolutions for $S O_{2 \ell+1} \times G L_{n}$ : Local theory, preprint.

[31] D. SOUDRY, On the archimedean theory of Rankin-Selberg Convolutions for $S O_{2 \ell+1} \times G L_{n}$, (Ann. Scient. Éc. Norm. Sup., Vol. 28, 1995, pp. 161-224).

[32] B. SPEH, Some results on principal series for $G L(n, \mathbb{R})$, Ph.D. Dissertation, (Massachusetts Institute of Technology, June, 1977).

[33] B. SPEH and D. VouAn, Reducibility of generalized principal series representations, (Acta Math., Vol. 145, 1980, pp. 227-299).

[34] M. TADIĆ, On regular square integrable representations of $p$-adic groups, Amer. J. Math., Vol. 120, 1997, pp. $159-210$.

[35] M. TADIĆ, Construction of square integrable representations of classical p-adic groups, (Mathematica Gottingensis Schriftenreihe des Sonderforschungsbereichs Geometrie und Analysis, Heft, Vol. 11, 1993, pp. 1-48.20).

[36] M. TADIĆ, Square integrable representations of classical p-adic groups of segment type, (Mathematica Gottingensis Schriftenreihe des Sonderforschungsbereichs Geometrie und Analysis, Heft, Vol. 15, 1994, pp. 1-16).

[37] D. VoGan, Gelfand-Kirillov dimension for Harish-Chandra modules, (Invent. Math., Vol. 48, 1978, pp. 75-98).

[38] D. VoGAN, Unitarizability of certain series of representations, (Ann. of Math., Vol. 120, 1984, pp. 141-187).

[39] D. Vogan, Representations of real reductive Lie groups, Birkhauser, Boston, 1981.

[40] N.R. WALLACH, Asymptotic expansions of generalized matrix entries of representations of real reductive groups, in (Lie Group Representations I, Lecture Notes in Math., Vol. 1024, Springer-Verlag, 1983, pp. 287-369).

[41] A.V. ZELEVINSKY, Induced representations of reductive p-adic groups II, on irreducible representations of $G L(n)$, (Ann. Scient. Éc. Norm. Sup., Vol. 13, 1980, pp. 165-210).

[42] G. Muí, Some results on square integrable representations; Irreducibility of standard representations, preprint.

[43] Y. ZHANG, The holomorphy and nonvanishing of normalized local intertwining operators, (Pacific J. Math., Vol. 180, 1997, pp. 385-398).

(Manuscript received July 20, 1997;

revised January 22, 1998.)

W. Casselman

University of British Columbia

Vancouver, British Columbia V6T 1Z2, Canada.

F SHAHIDI

Purdue University

West Lafayette, Indiana, 47907, USA. 